\title{
Quasi-periodic Solutions of Wave Equations with the Nonlinear Term Depending on the Time and Space Variables
}

\author{
Yi Wang* and Jie Rui
}

\begin{abstract}
This article is devoted to the study of a wave equation with a constant potential and an $x$-periodic and $t$-quasi-periodic nonlinear term subject to periodic boundary conditions. It is proved that the equation admits small amplitude, linear stable and $t$-quasi-periodic solutions for any constant potential and most frequency vectors.
\end{abstract}

\section{Introduction}

In this paper, a wave equation with an $x$-periodic and $t$-quasi-periodic nonlinear term

$$
u_{t t}-u_{x x}+\mu u+\varepsilon g(\omega t, x) u^{3}=0, \quad x \in[0,2 \pi]
$$

under periodic boundary conditions

$$
u(t, x)=u(t, x+2 \pi)
$$

is considered, where $\mu>0 ; \varepsilon$ is a small positive parameter; $m \geq 2$ is an integer; $\omega=$ $\left(\omega_{1}, \omega_{2}, \ldots, \omega_{m}\right) \in[\varrho, 2 \varrho]^{m}$ is a frequency vector with $\varrho>0$; and the function $g(\omega t, x)=$ $g(\vartheta, x),(\vartheta, x) \in \mathbb{T}^{m} \times[0,2 \pi]$, is real analytic in $(\vartheta, x)$ and quasi-periodic in $t$. We aim to explore that whether these equations have analytic and linearly stable quasi-periodic solutions.

The existence of quasi-periodic solutions for nonlinear wave equation has been studied by many authors. Currently there are relatively less method to find a quasi-periodic solution of nonlinear wave equations. Since the quasi-periodic solutions constructed via the KAM method can provide some dynamics information, such as linearly stability and zero Lyapunov exponent, we apply the KAM method in this paper.

Received August 8, 2018; Accepted June 30, 2019.

Communicated by Cheng-Hsiung Hsu.

2010 Mathematics Subject Classification. 70K43, 70K45, 70K40, 37K55.

Key words and phrases. quasi-periodic solutions, normal form, quasi-periodically forced nonlinear wave equation, $x$-dependent term, KAM for infinite-dimensional systems.

This work was partially supported by National Natural Science Foundation of China (Grant Nos. 11601270, 11501571).

*Corresponding author. 
In the 90's, the KAM theory has been successfully extended to infinite dimensional settings, including the wave equations. In [8,11, 15], the KAM theory has been generalized to prove the existence of quasi-periodic solutions for the nonlinear wave equations subject to Dirichlet or Neumann boundary conditions. Chierchia and You [4] showed that nonlinear wave equations with periodic boundary conditions admit quasi-periodic solutions. Yuan [16] considered the existence of quasi-periodic solutions of the completely resonant nonlinear wave equation. In [17, he proved there are many quasi-periodic solutions for the nonlinear wave equations with a prescribed potential. Bambusi, Berti, and Magistrelli [1] dealt with degenerate KAM theory for lower dimensional elliptic tori of infinite dimensional Hamiltonian system. An application to nonlinear wave equation was given in [1]. Geng and Ren [7] proved that the nonlinear wave equation admits quasi-periodic solutions with $b$-dimensional Diophantine frequencies. In particular, these Diophantine frequencies are the small dilation of a prescribed Diophantine vector. Zhang and Si [18], and Si [13] proved existence of quasi-periodic solutions for non-autonomous, quasi-periodically forced nonlinear wave equations. Berti, Biasco, and Procesi [2] considered the existence and stability of quasi-periodic solutions for reversible derivative wave equations. Gao, Zhang, and Ji [6] obtained the existence of quasi-periodic solutions for nonlinear wave equation under the general boundary conditions.

For the research about the existence of quasi-periodic solutions for nonlinear wave equations, Berti and Bolle [3] considered a wave equation with a quasi-periodic-in-time nonlinearity. In [3], the condition $\operatorname{ker}(-\Delta+V(x))=\{0\}$ was supposed and they obtained the $\mathbb{C}^{\infty}$ solutions not analytic solutions, where $V$ is the potential. Montalto 9], Corsi and Montalto [5] researched forced Kirchoff equations

$$
\partial_{t t} v-\left(1+\int_{\mathbb{T}^{d}}|\nabla v|^{2} d x\right) \Delta v=\delta f(\omega t, x)
$$

with periodic boundary conditions, where $\delta>0$ is a small parameter, $\omega$ is a parameter vector, $f$ is a sufficiently smooth function with zero average, and $f$ is not dependent of $v$. The existence of quasi-periodic solutions were proved and the problem of multiple eigenvalues is overcome. The proofs in [3, 5, 9] are based on Nash-Moser iterative schemes not the KAM method. In this paper, we suppose that the potential is a positive constant, and the nonlinear term $u^{3}$ depends on $\omega t$ and $x$. We use the KAM method to study the wave equation, find out real analytic quasi-periodic solutions, and give the expression of solutions.

Factually, in order to use the KAM iteration, it is required to transform the Hamiltonian into its Birkhoff normal form. To find real analytic quasi-periodic solutions, this symplectic transformation should be real analytic. This transformation can be constructed through the time-1-map of the flow of a Hamiltonian vector-field. 
Unfortunately, the Hamiltonian contains small divisors. It is necessary to ensure that the measure of parameters making the denominators zero is small. Since the nonlinear term depends on the space variable, one must estimate the measure of infinitely many small divisors. The reference [2] remarked that when the nonlinear term of a PDE is $x$-independent, "all the monomials of the corresponding vector field $X$ have momentum equal to zero". In [2], it is introduced a weighted majorant norm for vector fields to overcome the "non-conservation of the momentum" problem. While in this paper, we only assume $g$ is bounded.

To overcome the multiple spectrum problem, we construct the even solutions and separately calculate the 0th-eigenvalue part. But the estimate of measure is still meticulous and complex, which is the main work of this paper. In addition, since the equation is forced, the symplectic transformation is variant-coefficient. It is difficult to prove the regularity of the symplectic transformation. We introduce a symmetry integer set, divide the vector-field into the mean value part and the other part, and deal with the two parts in different ways.

The paper is organized as follows. In Section 2, we give the main result and transform the equation to an infinite dimensional Hamiltonian system. Section 3 is devoted to a Birkhoff normal form. In Section 4, we state a KAM theorem for nonlinear partial differential equations. Using this theorem, we complete the proof of our main theorem. Two lemmas are proved in the last section.

\section{Main result and Hamiltonian setting}

For $\varepsilon=0$, the equation 1.1 becomes

$$
u_{t t}-u_{x x}+\mu u=0
$$

The operator $A=-\frac{d^{2}}{d x^{2}}+\mu$ with periodic boundary conditions has eigenvalues $\left\{\lambda_{j}=\right.$ $\left.j^{2}+\mu, j \in \mathbb{Z}\right\}$ and the corresponding eigenfunctions

$$
\phi_{j}(x)= \begin{cases}\frac{1}{\sqrt{\pi}} \cos j x & \text { if } j>0, \\ \frac{1}{\sqrt{\pi}} \sin j x & \text { if } j<0, \\ \frac{1}{\sqrt{2 \pi}} & \text { if } j=0 .\end{cases}
$$

Since the eigenfunctions $\phi_{j}(x)(j \geq 0)$ are a completely orthogonal basis of the subspace consisting of all even functions of $L^{2}[0,2 \pi]$, we restrict ourselves to find some solutions which are even in $x$ so that we can avoid the trouble caused by the double eigenvalues.

Every solution of the linear wave equation (2.1) can be written as a super-position of 
the basic modes $\phi_{j}$, namely, for any subset $\mathcal{J}$ of $\mathbb{Z}$,

$$
u(t, x)=\sum_{j \in \mathcal{J}} q_{j}(t) \phi_{j}(x), \quad q_{j}(t)=I_{j} \cos \left(\sqrt{\lambda_{j}} t+\varphi_{j}^{0}\right)
$$

with amplitudes $I_{j} \geq 0$ and initial phases $\varphi_{j}^{0}$.

We first make some assumptions and give some notations. Define $D_{1}\left(\sigma_{1}\right):=\{\vartheta \mid$ $\left.|\operatorname{Im} \vartheta|<\sigma_{1}\right\}, D_{2}(2 a):=\{x|| \operatorname{Im} x \mid<2 a\},|g(\vartheta, x)|_{D_{1}\left(\sigma_{1}\right) \times D_{2}(2 a)}:=\sup _{(\vartheta, x) \in D_{1}\left(\sigma_{1}\right) \times D_{2}(2 a)}$ $|g(\vartheta, x)|$ and $|g(\vartheta, x)|_{D_{2}(2 a)}:=\sup _{x \in D_{2}(2 a)}|g(\vartheta, x)|$ for every fixed $\vartheta \in D_{1}\left(\sigma_{1}\right)$. Throughout this paper, we suppose that

$\left(\mathrm{H}_{1}\right) g$ even extends to a $2 \pi$-periodic function in $x$ whose Fourier series satisfies $g(\vartheta, x)=$ $g_{0}+\sum_{k \in \mathbb{Z}^{m} \backslash\{0\}} g_{k}(x) e^{\mathrm{i}\langle k, \vartheta\rangle}, 0 \neq g_{0} \in \mathbb{R}$, where $\mathrm{i}=\sqrt{-1}$ and $\langle\cdot, \cdot\rangle$ is the standard inner product in $\mathbb{C}^{m}$.

$\left(\mathrm{H}_{2}\right)$ For some $\sigma_{1}>0$ and $a>0, g$ analytically in $\vartheta, x$ extends to the domain $D_{1}\left(\sigma_{1}\right) \times$ $D_{2}(2 a)$ and $g$ is bounded in $D_{1}\left(\sigma_{1}\right) \times D_{2}(2 a)$ with finite norm $|g(\vartheta, x)|_{D_{1}\left(\sigma_{1}\right) \times D_{2}(2 a)}$.

Theorem 2.1. Assume $\left(\mathrm{H}_{1}\right)$ and $\left(\mathrm{H}_{2}\right)$ hold. For each $1 \leq n<\infty$ and each index set $\mathcal{J}=\left\{j_{0}<j_{1}<j_{2}<\cdots<j_{n}\right\} \subset \mathbb{N}:=\{0,1,2, \ldots\}$ satisfying $\min _{0 \leq i<n}\left(j_{i+1}-j_{i}\right) \leq n$ for any $\mu>0$ and for any $0<\varepsilon<\varepsilon^{*}$, where $\varepsilon^{*}$ is sufficiently small, there exist a subset $\Omega \subset[\varrho, 2 \varrho]^{m}$ with meas $\Omega>0$ and a subset $\Sigma_{\varepsilon} \subset \Sigma:=\Omega \times[0,1]^{n+1}$ with meas $\left(\Sigma \backslash \Sigma_{\varepsilon}\right)<\varepsilon^{1 / 3}$ which satisfies that for any $\xi=\left(\omega_{1}, \omega_{2}, \ldots, \omega_{m}, \widetilde{\xi}_{j_{0}}, \widetilde{\xi}_{j_{1}}, \ldots, \widetilde{\xi}_{j_{n}}\right) \in \Sigma_{\varepsilon}$, the boundary value problem (1.1) - 1.2 has a quasi-periodic and even solution in $x$. Furthermore, if $j_{0}=0$,

$u(t, x)=\sqrt{\frac{\varepsilon \widetilde{\xi}_{j_{0}}+\mathcal{O}\left(\varepsilon^{8 / 3}\right)}{\pi \sqrt{\mu}}} \cos \widetilde{\omega}_{j_{0}} t+\sum_{j_{i} \in \mathcal{J} \backslash\left\{j_{0}\right\}} \sqrt{\frac{2\left(\varepsilon \widetilde{\xi}_{j_{i}}+\mathcal{O}\left(\varepsilon^{8 / 3}\right)\right)}{\pi \sqrt{\lambda_{j_{i}}}}} \cos \widetilde{\omega}_{j_{i}} t \cos j_{i} x+\mathcal{O}\left(\varepsilon^{5 / 3}\right)$, and if $j_{0}>0$,

$$
u(t, x)=\sum_{j_{i} \in \mathcal{J}} \sqrt{\frac{2\left(\varepsilon \widetilde{\xi}_{j_{i}}+\mathcal{O}\left(\varepsilon^{8 / 3}\right)\right)}{\pi \sqrt{\lambda_{j_{i}}}}} \cos \widetilde{\omega}_{j_{i}} t \cos j_{i} x+\mathcal{O}\left(\varepsilon^{5 / 3}\right),
$$

where

$$
\left|\widetilde{\omega}_{j_{i}}(\xi)-\sqrt{\lambda_{j_{i}}}\right|<c \varepsilon^{2} \quad \text { for all } j_{i} \in \mathcal{J}
$$

Remark 2.2. The equation (1.1) has a large Cantor family of rotational $(m+n+1)$ dimensional tori with frequency vectors $\widetilde{\omega}$, where $\widetilde{\omega}=\left(\omega_{1}, \omega_{2}, \ldots, \omega_{m}, \widetilde{\omega}_{j_{0}}, \widetilde{\omega}_{j_{1}}, \ldots, \widetilde{\omega}_{j_{n}}\right)$.

Remark 2.3. The function $g$ can be described as an analytic even function, which is periodic in $x$ and quasi-periodic in $t$. Moreover, the mean value of $g$ is not zero. For example, $g(x, t)=2+\cos x(\cos t+\cos \sqrt{3} t)$. 
Remark 2.4. To avoid the double eigenvalues, we restrict ourselves to choose an even complete orthogonal basis $\phi_{j}(x)(j \geq 0)$, which leads to the even solution.

Remark 2.5. The function $g$ can be defined in any strip $D_{2}(\widehat{a})$ as long as $\widehat{a}>a$. The fixed positive $a$ is used to define the Hilbert space $l^{a, s}$ below.

Remark 2.6. Theorem 2.1 holds true for $m=1$.

We comment on the result as follows:

1. $x$-dependence. The nonlinearity $g$ in 1.1 can explicitly contain the space variable $x$, which implies that this equation is variant under $x$ translations. Thus the socalled "compact form" condition or "zero momentum" condition are not satisfied. The measure estimate is more complex than that in $[2,7,13,16,18$. The decay property plays an important role, e.g. 2.6.

2. quasi-periodical forcing. The equation (1.1) is quasi-periodically forced. So a quasi-periodic symplectic transform is required (see the proof of Proposition 3.6), which can transform the Hamiltonian into its normal form.

3. The frequency $\widetilde{\omega}$ of the solution. Since extra parameters are added in the proof, we obtain quasi-periodic solutions with additional frequencies but not those with the same frequency $\omega$ as $g$.

4. The parameter $\mu$. The result holds true for all $\mu>0$. In addition, the discussion about the completely resonant wave equations (namely $\mu=0$ ) may be more complex.

In this paper, $C$ denotes a universal constant if we do not care its value. We rewrite the wave equation (1.1) as follows:

$$
\partial_{t} u=v, \quad \partial_{t} v+A u=-\varepsilon g(\omega t, x) u^{3},
$$

where $A=-d^{2} / d x^{2}+\mu, t \in \mathbb{R}$. It is well known that the equation $(2.2)$ can be studied as an infinite dimensional Hamiltonian system with coordinates $u$ and $v=\partial_{t} u$. The Hamiltonian for $(2.2)$ is then

$$
H=\frac{1}{2}(v, v)+\frac{1}{2}(A u, u)+\varepsilon \int_{0}^{2 \pi} \chi(u, x, \omega t) d x,
$$

where $\chi(u, x, \omega t)=\frac{1}{4} g(\omega t, x) u^{4}$, and $(\cdot, \cdot)$ denotes the scalar product in $L^{2}[0,2 \pi]$.

We introduce the coordinates $q=\left(q_{0}, q_{1}, q_{2}, \ldots\right)$ and $p=\left(p_{0}, p_{1}, p_{2}, \ldots\right)$ by setting

$$
u(t, x)=\sum_{j \geq 0} \frac{q_{j}(t)}{\sqrt[4]{\lambda_{j}}} \phi_{j}(x), \quad v(t, x)=\sum_{j \geq 0} \sqrt[4]{\lambda_{j}} p_{j}(t) \phi_{j}(x) .
$$


The coordinates are taken from some Hilbert space $l^{a, s}(s>1 / 2)$ of all real valued infinite sequences

$$
\begin{aligned}
l^{a, s} & =l^{a, s}(\mathbb{R}) \\
& :=\left\{q=\left(q_{0}, q_{1}, q_{2}, \ldots\right), q_{i} \in \mathbb{R}, i \geq 0 \text { s.t. }\left(\|q\|_{a, s}\right)^{2}=\left|q_{0}\right|^{2}+\sum_{i \geq 1}\left|q_{i}\right|^{2} i^{2 s} e^{2 a i}<\infty\right\} .
\end{aligned}
$$

Thus, we obtain the Hamiltonian $H=\Lambda+\varepsilon G$, where

$$
\Lambda=\frac{1}{2} \sum_{j \geq 0} \sqrt{\lambda_{j}}\left(q_{j}^{2}+p_{j}^{2}\right) \quad \text { and } \quad G=\frac{1}{4} \int_{0}^{2 \pi} g(\omega t, x)\left(\sum_{j \geq 0} \frac{q_{j}(t)}{\sqrt[4]{\lambda_{j}}} \phi_{j}(x)\right)^{4} d x
$$

The corresponding equations of motion are

$$
\dot{q}_{j}=\frac{\partial H}{\partial p_{j}}=\sqrt{\lambda_{j}} p_{j}, \quad \dot{p}_{j}=-\frac{\partial H}{\partial q_{j}}=-\sqrt{\lambda_{j}} q_{j}-\varepsilon \frac{\partial G}{\partial q_{j}}, \quad j \geq 0
$$

with respect to the symplectic structure $\sum d q_{i} \wedge d p_{i}$ on $l^{a, s} \times l^{a, s}$. According to [11, 13], $u$ is a classical solution of (1.1) and the following lemma holds true.

Lemma 2.7. If $I \subseteq \mathbb{R}$ is an interval and a curve $I \rightarrow(p(t), q(t))$ is a real analytic solution of $(2.3)$, then

$$
u(t, x)=\sum_{j \geq 0} \frac{q_{j}(t)}{\sqrt[4]{\lambda_{j}}} \phi_{j}(x)
$$

is a classical solution of 1 that is real analytic on $I \times[0,2 \pi]$.

By introducing a pair of action-angle variables $(J, \vartheta) \in \mathbb{R}^{m} \times \mathbb{T}^{m}\left(\mathbb{T}^{m}:=\mathbb{R}^{m} / 2 \pi \mathbb{Z}^{m}\right)$, one can obtain an equivalent Hamiltonian that does not depend on the time variable $t$. So an autonomous formulation of our problem is reached as follows:

$$
\dot{q}_{j}=\frac{\partial H}{\partial p_{j}}, \quad \dot{p}_{j}=-\frac{\partial H}{\partial q_{j}}, \quad j \geq 0, \quad \dot{\vartheta}=\omega, \quad \dot{J}=-\varepsilon \frac{\partial G}{\partial \vartheta}=-\varepsilon \frac{\partial \int_{0}^{2 \pi} \chi d x}{\partial \vartheta},
$$

which is a Hamiltonian system (with respect to the symplectic structure $d \vartheta \wedge d J+\sum d q_{i} \wedge$ $d p_{i}$ ) with the Hamiltonian

$$
H=\langle\omega, J\rangle+\frac{1}{2} \sum_{j \geq 0} \sqrt{\lambda_{j}}\left(q_{j}^{2}+p_{j}^{2}\right)+\varepsilon G(q, \vartheta)
$$

where $\langle\cdot, \cdot\rangle$ is the standard inner product in $\mathbb{C}^{m}$.

To continue our investigation for the Hamiltonian (2.4), we need to establish the regularity of the nonlinear Hamiltonian vector field $X_{G}$ associated to $G$. Let $l_{b}^{2}$ and $L^{2}$, 
respectively, be the Hilbert spaces of all bi-infinite, square summated sequences with complex coefficients and all square-integrable complex-valued functions on $[0,2 \pi]$. Suppose that

$$
\mathcal{F}: l_{b}^{2} \rightarrow L^{2}, \quad q \mapsto \mathcal{F} q=\frac{1}{\sqrt{2 \pi}} \sum_{j} q_{j} e^{\mathrm{i} j x}
$$

is the inverse discrete Fourier transform, which defines an isometry between the two spaces. The subspaces $l_{b}^{a, s} \subset l_{b}^{2}$ consist, by definition, of all bi-infinite sequences with the finite form

$$
\left(\|q\|_{a, s}^{1}\right)^{2}=\left|q_{0}\right|^{2}+\sum_{i}\left|q_{i}\right|^{2}|i|^{2 s} e^{2 a|i|} .
$$

Through $\mathcal{F}$ they define subspaces $W^{a, s}[0,2 \pi] \subset L^{2}[0,2 \pi]$ that are normed by setting $\|\mathcal{F} q\|_{a, s}^{1}=\|q\|_{a, s}^{1}$.

The following lemma has been proven in 11 and we only give the result.

Lemma 2.8. The space $l_{b}^{a, s}$ is a Hilbert algebra with respect to convolution of the sequences $(q * p)_{j}:=\sum_{k} q_{j-k} p_{k}$, and

$$
\|q * p\|_{a, s}^{1} \leq c\|q\|_{a, s}^{1}\|p\|_{a, s}^{1},
$$

where the constant $c$ depends only on s. Consequently, $W^{a, s}$ is a Hilbert algebra with respect to multiplication of functions.

Using the above lemma, we prove the following result.

Lemma 2.9. The gradient $\partial_{q} G$ is real analytic as a map from some neighborhood of origin in $l^{a, s}$ into $l^{a, s+1}$, with $\left\|\partial_{q} G\right\|_{a, s+1}=\mathcal{O}\left(\|q\|_{a, s}\right)^{3}$.

Proof. Let $q \in l^{a, s}$. Consider as a function on $[0,2 \pi], u=\sum_{j \geq 0} \frac{q_{j}(t)}{\sqrt[4]{\lambda_{j}}} \phi_{j}(x)$ is in $W^{a, s+1 / 2}$ with

$$
\|u\|_{a, s+1 / 2}^{1} \leq c\|q\|_{a, s} .
$$

For every $\vartheta \in D_{1}\left(\sigma_{1}\right)$, we expand $g$ in a Fourier series $g(\vartheta, x)=\frac{1}{\sqrt{2 \pi}} \sum_{j} g_{j}(\vartheta) e^{\mathrm{i} j x}$. By using of Lemma A.1 in [12], for every $j \in \mathbb{Z}$,

$$
\left|g_{j}(\vartheta)\right| \leq C|g(\vartheta, x)|_{D_{2}(2 a)} e^{-2 a|j|}
$$

holds. So

$$
\begin{aligned}
\left(\|g\|_{a, s+1 / 2}^{1}\right)^{2} & =\left|g_{0}(\vartheta)\right|^{2}+\sum_{j \neq 0}\left|g_{j}(\vartheta)\right|^{2}|j|^{2 s+1} e^{2 a|j|} \\
& <C \sum|g(\vartheta, x)|_{D_{2}(2 a)}^{2} e^{-4 a|j|}[j]^{2 s+1} e^{2 a|j|} \\
& <C|g(\vartheta, x)|_{D_{1}\left(\sigma_{1}\right) \times D_{2}(2 a)}^{2} \sum_{j} e^{-2 a|j|}[j]^{2 s+1}<\infty .
\end{aligned}
$$


Therefore, $g \in W^{a, s+1 / 2}[0,2 \pi]$. It follows from (2.5) that, by Lemma 2.8 and the analyticity of $g$ and $u^{3}$, the function $g(\vartheta, x) u^{3}$ also belongs to $W^{a, s+1 / 2}[0,2 \pi]$ with $\left\|g(\vartheta, x) u^{3}\right\|_{a, s+1 / 2}^{1}$ $\leq C\left(\|q\|_{a, s}\right)^{3}$ in a sufficiently small neighborhood of the origin, where $C$ depends on $s, a$, $\sigma_{1}$ and $g$. On the other hand, since

$$
\frac{\partial G}{\partial q_{j}}=\frac{1}{\sqrt[4]{\lambda_{j}}} \int_{0}^{2 \pi} g(\vartheta, x) u^{3} \phi_{j}(x) d x
$$

the components of $G_{q}$ are the Fourier coefficients of $g(\vartheta, x) u^{3}$, so $G_{q}$ belongs to $l^{a, s}$, with

$$
\left\|G_{q}\right\|_{a, s+1} \leq C\left\|g(\vartheta, x) u^{3}\right\|_{a, s+1 / 2}^{1} \leq C\left(\|q\|_{a, s}\right)^{3},
$$

where the last $C$ depends on $s, a, \mu, \sigma_{1}$ and $g$. The regularity of $G_{q}$ follows from the regularity of its component and its local boundedness.

\section{Partial Birkhoff normal form}

Since $\chi(u, x, \vartheta)=\frac{1}{4} g(\vartheta, x) u^{4}$ and $u=\sum_{j} \frac{q_{j}}{\sqrt[4]{\lambda_{j}}} \phi_{j}$, we find that

$$
G(q, \vartheta)=\frac{1}{4} \sum_{i, j, d, l} \frac{1}{\sqrt[4]{\lambda_{i} \lambda_{j} \lambda_{d} \lambda_{l}}} \int_{0}^{2 \pi} g(\vartheta, x) \phi_{i} \phi_{j} \phi_{d} \phi_{l} d x q_{i} q_{j} q_{d} q_{l}
$$

From $\left(\mathrm{H}_{1}\right)$,

$$
G(q, \vartheta)=\frac{1}{4} \sum_{i, j, d, l} \frac{G_{i j d l}}{\sqrt[4]{\lambda_{i} \lambda_{j} \lambda_{d} \lambda_{l}}} q_{i} q_{j} q_{d} q_{l}+\frac{1}{4} \sum_{|k| \geq 1, i, j, d, l} \frac{G_{k, i j d l}}{\sqrt[4]{\lambda_{i} \lambda_{j} \lambda_{d} \lambda_{l}}} e^{\mathrm{i}\langle k, \vartheta\rangle} q_{i} q_{j} q_{d} q_{l},
$$

where

$$
G_{i j d l}=g_{0} \int_{0}^{2 \pi} \phi_{i} \phi_{j} \phi_{d} \phi_{l} d x
$$

and

$$
G_{k, i j d l}=\int_{0}^{2 \pi} g_{k}(x) \phi_{i} \phi_{j} \phi_{d} \phi_{l} d x, \quad|k| \geq 1
$$

An easy computation shows that $G_{i j d l}=0$ unless $i \pm j \pm d \pm l=0$ for at least one combination of plus and minus signs. In particular, we have

$$
G_{i j i j}=\frac{g_{0}}{4 \pi}\left(2+\delta_{i j}\right) \quad \text { where } \delta_{i j}= \begin{cases}1 & \text { if } i=j \neq 0 \\ 0 & \text { if } i=j=0 \\ 0 & \text { if } i \neq j\end{cases}
$$


We switch to the complex coordinates

$$
z_{i}=\frac{q_{i}+\mathrm{i} p_{i}}{\sqrt{2}}, \quad \bar{z}_{i}=\frac{q_{i}-\mathrm{i} p_{i}}{\sqrt{2}}
$$

that live in the now complex Hilbert space $l^{a, s}=l^{a, s}(\mathbb{C})$. Then, the Hamiltonian (with respect to the symplectic structure $\left.d \vartheta \wedge d J+\mathrm{i} \sum_{i} d z_{i} \wedge d \bar{z}_{i}\right)$ is given by

$$
H=\Lambda+\varepsilon G,
$$

where

$$
\Lambda=\langle\omega, J\rangle+\sum_{j} \sqrt{\lambda_{j}} z_{j} \bar{z}_{j}, \quad G=\frac{1}{4} \int_{0}^{2 \pi} g(\vartheta, x)\left(\sum_{j \geq 0} \frac{z_{j}+\bar{z}_{j}}{\sqrt[4]{4 \lambda_{j}}} \phi_{j}(x)\right)^{4} d x .
$$

Moreover, from (3.1), 3.2$)$ and $(3.3)$, we have

$$
\begin{aligned}
G= & \frac{1}{16} \sum_{i, j, d, l, i \pm j \pm d \pm l=0} \frac{G_{i j d l}}{\sqrt[4]{\lambda_{i} \lambda_{j} \lambda_{d} \lambda_{l}}}\left(z_{i}+\bar{z}_{i}\right)\left(z_{j}+\bar{z}_{j}\right)\left(z_{d}+\bar{z}_{d}\right)\left(z_{l}+\bar{z}_{l}\right) \\
& +\frac{1}{16} \sum_{|k| \geq 1, i, j, d, l} \frac{G_{k, i j d l}}{\sqrt[4]{\lambda_{i} \lambda_{j} \lambda_{d} \lambda_{l}}} e^{\mathrm{i}\langle k, \vartheta\rangle}\left(z_{i}+\bar{z}_{i}\right)\left(z_{j}+\bar{z}_{j}\right)\left(z_{d}+\bar{z}_{d}\right)\left(z_{l}+\bar{z}_{l}\right) .
\end{aligned}
$$

Next we transform the Hamiltonian (3.5) into some partial Birkhoff form of order four so that it may serve as a small perturbation of some nonlinear integrable system in a sufficiently small neighborhood of the origin.

Assume $\mathbb{Z}^{\prime}=\{\ldots,-2,-1,-0,0,1,2, \ldots\}$ and $1 \leq n<\infty$ fixed. Let $\mathcal{L}_{n}=\{(i, j, d, l) \in$ $\left.\mathbb{Z}^{4}: \min (|i|,|j|,|d|,|l|) \leq n\right\}$ and $\mathcal{N}_{n} \subset \mathcal{L}_{n}$ be the subset of all $(i, j, d, l) \equiv(p,-p, q,-q)$. That is, they are of the form $(p,-p, q,-q)$ or some permutation of it. We write

$$
\lambda_{i}^{\prime}:=\operatorname{sgn} i \cdot \sqrt{\lambda_{|i|}} \text {. }
$$

Remark 3.1. In the set $\mathbb{Z}^{\prime}$, we set 0 and -0 to be two different indices, which can prevent $z_{0}$ and $\bar{z}_{0}$ from applying the same coordinate symbol in the proof of Proposition 3.6.

The following lemmas are necessary and important. We use them to prove Proposition 3.6. The proofs of Lemmas 3.3 and 3.5 can be found in [14]. The proofs of Lemmas 3.2 and 3.4 are shown in Appendix.

Lemma 3.2. If $(i, j, d, l) \in \mathcal{L}_{n} / \mathcal{N}_{n}$ and

$$
|i| \pm|j| \pm|d| \pm|l|=0
$$

then there exists a constant $c$ depending on $\mu$ and $n$ such that

$$
\left|\lambda_{i}^{\prime}+\lambda_{j}^{\prime}+\lambda_{d}^{\prime}+\lambda_{l}^{\prime}\right| \geq c>0
$$

holds for every compact $\mu$-interval in $(0, \infty)$. 
Lemma 3.3. There is a subset $\underline{\Omega} \subset[\varrho, 2 \varrho]^{m}$ such that every $\omega \in \underline{\Omega}$ satisfies that

$$
|\langle k, \omega\rangle| \geq \frac{\varrho \varepsilon^{1 / 3}}{|k|^{m+1}} \quad \text { for all } 0 \neq k \in \mathbb{Z}^{m}
$$

and meas $\underline{\Omega} \geq\left(1-C_{1} \varepsilon^{1 / 3}\right) \varrho^{m}$, where the constant $C_{1}$ depends on $m$ and meas denotes Lebesgue measure for sets.

Lemma 3.4. Assume that $(i, j, d, l) \in \mathcal{L}_{n}$ and $k \neq 0$. Then, when $\varepsilon$ is small enough, there is a subset $\bar{\Omega} \subset[\varrho, 2 \varrho]^{m}$ satisfying that, for any $\omega \in \bar{\Omega}$ and $\lambda_{i}^{\prime}+\lambda_{j}^{\prime}+\lambda_{d}^{\prime}+\lambda_{l}^{\prime} \neq 0$,

$$
\left|\lambda_{i}^{\prime}+\lambda_{j}^{\prime}+\lambda_{d}^{\prime}+\lambda_{l}^{\prime}+\langle k, \omega\rangle\right| \geq \frac{\varrho \varepsilon^{1 / 3}}{|k|^{m+3}} .
$$

Moreover, meas $\bar{\Omega} \geq \varrho^{m}\left(1-C_{2} \varepsilon^{1 / 3}\right)$, where $C_{2}$ is a constant depending on $n, m, \varrho$ and $\mu$.

Lemma 3.5. For $x_{k} \in \mathbb{C}$ and $k \in \mathbb{Z}^{m}$, if the series $\sum_{k}[k]^{2 m+1}\left|x_{k}\right|^{2}$ converges, then the inequality $\left|\sum_{k} x_{k}\right|^{2} \leq c \sum_{k}[k]^{2 m+1}\left|x_{k}\right|^{2}$ holds, where $[k]=\max \{|k|, 1\},|k|=\left|k_{1}\right|+\left|k_{2}\right|+$ $\cdots+\left|k_{m}\right|$ and $c$ is a constant depending on $m$.

Consider the Hamiltonian (3.5). For each fixed subset $\mathcal{L}_{n}$ and $\mu>0$, the following Proposition 3.6 holds. In the following, $\mathscr{A}\left(l^{a, s}, l^{a, \bar{s}}\right)$ denotes the class of all real analytic maps from some neighborhood of the origin in $l^{a, s}$ to $l^{a, \bar{s}}$.

Proposition 3.6. When $\varepsilon$ is small enough, there exists a subset $\Omega \subset[\varrho, 2 \varrho]^{m}$ with meas $\Omega>0$ and for every $\omega \in \Omega$, there is a real analytic, symplectic change of coordinates $\Psi$ which can transform the Hamiltonian (3.5) into its Birkhoff normal form, i.e.,

$$
H \circ \Psi=\Lambda+\varepsilon \bar{G}+\varepsilon \check{G}+\varepsilon^{2} K
$$

where $X_{\check{G}}, X_{\bar{G}}, X_{K} \in \mathcal{A}\left(l^{a, s}, l^{a, s+1}\right),|\check{G}|=\mathcal{O}\left(\left(\|Z\|_{a, s}\right)^{4}\right), \varepsilon^{1 / 3}|K|=\mathcal{O}\left(\left(\|z\|_{a, s}\right)^{6}\right)$ and

$$
\bar{G}(z, \bar{z})=\frac{1}{2} \sum_{\min \{i, j\} \leq n} \bar{G}_{i j}\left|z_{i}\right|^{2}\left|z_{j}\right|^{2}
$$

with uniquely determined coefficients

$$
\bar{G}_{i j}= \begin{cases}\frac{3 g_{0}}{4 \pi \sqrt{\lambda_{i} \lambda_{j}}} & \text { if } i \neq j, \\ \frac{9 g_{0}}{16 \pi \lambda_{i}} & \text { if } i=j \neq 0, \\ \frac{3 g_{0}}{8 \pi \lambda_{0}} & \text { if } i=j=0 .\end{cases}
$$

Here $Z=\left(z_{n+1}, z_{n+2}, \ldots\right)$ and $z=\left(z_{0}, z_{1}, z_{2}, \ldots\right)$. Moreover, the transformation $\Psi$ is defined in a complex neighborhood $D_{1}\left(\sigma_{1} / 2\right):=\left\{\vartheta|| \operatorname{Im} \vartheta \mid<\sigma_{1} / 2\right\}$ of the tour $\mathbb{T}^{m}$ and a neighborhood of the origin in $l^{a, s}$. 
Proof. For convenience, we introduce another coordinates $\left(\ldots, w_{-2}, w_{-1}, w_{-0}, w_{0}, w_{1}, w_{2}\right.$, $\ldots)$ in $\widehat{l}_{b}^{a, s}$ by letting $z_{0}=w_{0}, \bar{z}_{0}=w_{-0}, z_{j}=w_{j}, \bar{z}_{j}=w_{-j}(j \geq 1)$, where the space $\widehat{l}_{b}^{a, s}=\widehat{l}_{b}^{a, s}(\mathbb{C}):=\left\{\left(\ldots, w_{-1}, w_{-0}, w_{0}, w_{1}, \ldots\right), w_{i} \in \mathbb{C}\right.$ s.t. $\left.\left(\|w\|_{a, s}^{2}\right)^{2}<\infty\right\}$ and $\left(\|w\|_{a, s}^{2}\right)^{2}=$ $\left|w_{-0}\right|^{2}+\left|w_{0}\right|^{2}+\sum_{|i|=1}^{\infty}\left|w_{i}\right|^{2}|i|^{2 s} e^{2 a|i|}$. So we have

$$
\begin{aligned}
H= & \langle\omega, J\rangle+\sum_{j \geq 0} \sqrt{\lambda_{j}} w_{j} w_{-j}+\frac{\varepsilon}{16} \sum_{\substack{i, j, d, l \\
|i| \pm|j| \pm|d| \pm|l|=0}}^{\prime} g_{i j d l} w_{i} w_{j} w_{d} w_{l} \\
& +\frac{\varepsilon}{16} \sum_{|k| \geq 1} \sum_{i, j, d, l}^{\prime} g_{k, i j d l} e^{\mathrm{i}\langle k, \vartheta\rangle} w_{i} w_{j} w_{d} w_{l},
\end{aligned}
$$

where

$$
g_{i j d l}=\frac{G_{|i||j||d||l|}}{\sqrt[4]{\lambda_{|i|} \lambda_{|j|} \lambda_{|d|} \lambda_{|l|}}}, \quad g_{k, i j d l}=\frac{G_{k,|i||j||d||l|}}{\sqrt[4]{\lambda_{|i|} \lambda_{|j|} \lambda_{|d|} \lambda_{|l|}}},
$$

and the prime symbols indicate that the subscripted indices run through $\mathbb{Z}^{\prime}$. Since $G_{i j d l}=$ 0 unless $i \pm j \pm d \pm l=0$ for at least one combination of plus and minus signs,

$$
g_{i j d l}=0 \quad \text { unless }|i| \pm|j| \pm|d| \pm|l|=0
$$

Step 1. We construct the symplectic transformation. Consider a Hamiltonian function

$$
\mathcal{F}=\varepsilon F=\varepsilon \sum_{i, j, d, l}^{\prime} F_{i j d l} w_{i} w_{j} w_{d} w_{l}+\varepsilon \sum_{|k| \geq 1} \sum_{i, j, d, l}^{\prime} F_{k, i j d l} e^{\mathrm{i}\langle k, \vartheta\rangle} w_{i} w_{j} w_{d} w_{l}
$$

with coefficients

$$
\mathrm{i} F_{i j d l}= \begin{cases}\frac{g_{i j d l}}{16\left(\lambda_{i}^{\prime}+\lambda_{j}^{\prime}+\lambda_{d}^{\prime}+\lambda_{l}^{\prime}\right)} & \text { if }(i, j, d, l) \in \mathcal{L}_{n} \backslash \mathcal{N}_{n} \text { and }|i| \pm|j| \pm|d| \pm|l|=0 \\ 0 & \text { otherwise }\end{cases}
$$

and for $k \neq 0$,

$$
\mathrm{i} F_{k, i j d l}= \begin{cases}\frac{g_{k, i j d l}}{16\langle k, \omega\rangle} & \text { if }(i, j, d, l) \in \mathcal{L}_{n}, \lambda_{i}^{\prime}+\lambda_{j}^{\prime}+\lambda_{d}^{\prime}+\lambda_{l}^{\prime}=0 \text { and }|k| \geq 1, \\ \frac{g_{k, i j d l}}{16\left(\lambda_{i}^{\prime}+\lambda_{j}^{\prime}+\lambda_{d}^{\prime}+\lambda_{l}^{\prime}+\langle k, \omega\rangle\right)} & \text { if }(i, j, d, l) \in \mathcal{L}_{n}, \lambda_{i}^{\prime}+\lambda_{j}^{\prime}+\lambda_{d}^{\prime}+\lambda_{l}^{\prime} \neq 0 \text { and }|k| \geq 1, \\ 0 & \text { otherwise. }\end{cases}
$$

Let $\Psi=X_{\mathcal{F}}^{1}$ be the time-1 map of the vector field of the Hamiltonian $\mathcal{F}$. Expanding at $t=0$ and using Taylor's formula we obtain

$$
\begin{aligned}
H \circ \Psi & =H+\{H, \mathcal{F}\}+\int_{0}^{1}(1-t)\{\{H, \mathcal{F}\}, \mathcal{F}\} \circ X_{\mathcal{F}}^{t} d t \\
& =\Lambda+\varepsilon G+\varepsilon\{\Lambda, F\}+\varepsilon^{2}\{G, F\}+\varepsilon^{2} \int_{0}^{1}(1-t)\{\{H, F\}, F\} \circ X_{\mathcal{F}}^{t} d t .
\end{aligned}
$$


Now we compute $G+\{\Lambda, F\}$. If we let

$$
I_{i j d l}:=\left[\frac{1}{16} g_{i j d l}-\mathrm{i}\left(\lambda_{i}^{\prime}+\lambda_{j}^{\prime}+\lambda_{d}^{\prime}+\lambda_{l}^{\prime}\right) F_{i j d l}\right] w_{i} w_{j} w_{d} w_{l}
$$

and

$$
I_{k, i j d l}:=\left[\frac{1}{16} g_{k, i j d l}-\mathrm{i}\left(\lambda_{i}^{\prime}+\lambda_{j}^{\prime}+\lambda_{d}^{\prime}+\lambda_{l}^{\prime}+\langle k, \omega\rangle\right) F_{k, i j d l}\right] e^{\mathrm{i}\langle k, \vartheta\rangle} w_{i} w_{j} w_{d} w_{l},
$$

then, from 3.11,

$$
\begin{aligned}
& G+\{\Lambda, F\}=\sum_{\substack{i, j, d, l \\
|i| \pm|j| \pm|d| \pm|l|=0}}^{\prime} I_{i j d l}+\sum_{|k| \geq 1} \sum_{i, j, d, l}^{\prime} I_{k, i j d l}
\end{aligned}
$$

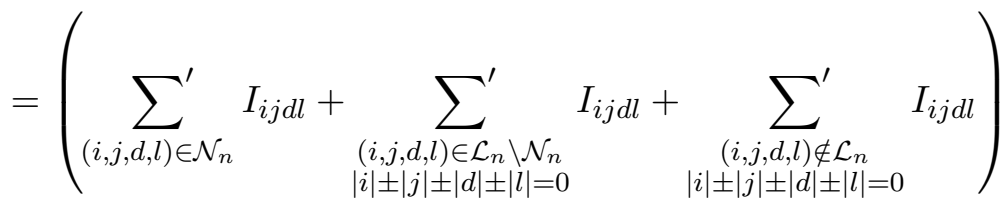

$$
\begin{aligned}
& +\left(\sum_{(i, j, d, l) \in \mathcal{L}_{n}}^{\prime} \sum_{|k| \geq 1} I_{k, i j d l}+\sum_{(i, j, d, l) \notin \mathcal{L}_{n}}^{\prime} \sum_{|k| \geq 1} I_{k, i j d l}\right) .
\end{aligned}
$$

By using of the definition of $F$, we have that

$$
\begin{gathered}
\sum_{\substack{(i, j, d, l) \in \mathcal{L}_{n} \backslash \mathcal{N}_{n} \\
|i| \pm|j| \pm|d| \pm|l|=0}}^{\prime} I_{i j d l}=\sum_{(i, j, d, l) \in \mathcal{L}_{n}}^{\prime} \sum_{|k| \geq 1} I_{k, i j d l}=0, \\
I_{i j d l}=\frac{1}{16} g_{i j d l} w_{i} w_{j} w_{d} w_{l}, \quad(i, j, d, l) \notin \mathcal{L}_{n}
\end{gathered}
$$

and

$$
I_{k, i j d l}=\frac{1}{16} g_{k, i j d l} e^{\mathrm{i}\langle k, \vartheta\rangle} w_{i} w_{j} w_{d} w_{l}, \quad(i, j, d, l) \notin \mathcal{L}_{n}, \quad|k| \geq 1
$$

hold. The last two equalities yield that

$$
\begin{aligned}
& \sum_{\substack{(i, j, d, l) \notin \mathcal{L}_{n} \\
|i| \pm|j| \pm|d| \pm|l|=0}}^{\prime} I_{i j d l}+\sum_{(i, j, d, l) \notin \mathcal{L}_{n}}^{\prime} \sum_{|k| \geq 1} I_{k, i j d l} \\
= & \sum_{\substack{(i, j, d, l) \notin \mathcal{L}_{n} \\
|k| \geq 1}}^{\prime} \sum_{|k|} \frac{1}{16} g_{k, i j d l} e^{i\langle k, \vartheta\rangle} w_{i} w_{j} w_{d} w_{l} \\
:= & \check{G}
\end{aligned}
$$

where we define $g_{0, i j d l}:=g_{i j d l}$. So, it derives from 3.13), 3.14) and 3.15) that

$$
G+\{\Lambda, F\}=\bar{G}+\check{G},
$$


where

$$
\bar{G}:=\sum_{(i, j, d, l) \in \mathcal{N}_{n}}^{\prime} I_{i j d l} .
$$

From (3.10) and (3.4), (3.8) and (3.9) are satisfied. Therefore, using (3.12) and (3.16),

$$
H \circ \Psi=\Lambda+\varepsilon \bar{G}+\varepsilon \check{G}+\varepsilon^{2}\{G, F\}+\varepsilon^{2} \int_{0}^{1}(1-t)\{\{H, F\}, F\} \circ X_{\mathcal{F}}^{t}
$$

holds true.

Step 2. We prove that $\Psi$ is real analytic.

Claim. The vector-field of the Hamiltonian $X_{F}$ is real analytic in a complex neighborhood $\vartheta \in D_{1}\left(\sigma_{1} / 2\right)$ of $\mathbb{T}^{m}$ and some neighborhood of the origin in $\widehat{l}_{b}^{a, s}$. Furthermore, it satisfies

$$
\left\|\frac{\partial F}{\partial w}\right\|_{a, s+1}^{2} \leq \frac{C}{\varepsilon^{2 / 3}}\left(\|w\|_{a, s}^{2}\right)^{3} .
$$

In fact, letting $w \in \widehat{l}_{b}^{a, s}, \vartheta \in D_{1}\left(\sigma_{1}\right)$, and from 3.2, 3.7) and 3.10), we have that

$$
\left|F_{r j d l}\right|=\left|\frac{g_{r j d l}}{16\left(\lambda_{r}^{\prime}+\lambda_{j}^{\prime}+\lambda_{d}^{\prime}+\lambda_{l}^{\prime}\right)}\right| \leq \frac{C\left|g_{0}\right|}{\left(\lambda_{r} \lambda_{j} \lambda_{d} \lambda_{l}\right)^{1 / 4}}
$$

holds for all $r, j, d, l \in \mathbb{Z}^{\prime}$, where the constant $C$ depends on $g, n$ and $\mu$. On the other hand, we can get the Fourier expansion of $g_{k}$, i.e.,

$$
g_{k}(x)=\sum_{\tau \in \mathbb{Z}} g_{k}^{\tau} e^{\mathrm{i} \tau x}
$$

So, fixing $r, j, d$ and $l \in \mathbb{Z}^{\prime}$, it follows from (3.3) that

$$
\begin{aligned}
G_{k,|r||j||d||l|} & =\sum_{\tau \in \mathbb{Z}} g_{k}^{\tau} \int_{0}^{2 \pi} e^{\mathrm{i} \tau x} \phi_{|r|} \phi_{|j|} \phi_{|d|} \phi_{|l|} d x \\
& =\sum_{\tau \in \mathbb{Z}} g_{k}^{\tau} \int_{0}^{2 \pi} \cos \tau x \phi_{|r|} \phi_{|j|} \phi_{|d|} \phi_{|l|} d x
\end{aligned}
$$

Easy computations show that

$$
\int_{0}^{2 \pi} \cos \tau x \phi_{|r|} \phi_{|j|} \phi_{|d|} \phi_{|l|} d x=0 \quad \text { unless } \tau \pm|r| \pm|j| \pm|d| \pm|l|=0
$$

holds for at least one combination of plus and minus signs and

$$
\left|\int_{0}^{2 \pi} \cos \tau x \phi_{|r|} \phi_{|j|} \phi_{|d|} \phi_{|l|} d x\right| \leq \frac{2}{\pi}
$$

holds for all $\tau \in \mathbb{Z}$ and $r, j, d, l \in \mathbb{Z}^{\prime}$. 
According to Lemmas 3.3 and 3.4 now we suppose that $\Omega=\bar{\Omega} \cap \underline{\Omega}$. It is obvious that meas $\Omega \geq \varrho^{m}\left(1-C_{1} \varepsilon^{1 / 3}-C_{2} \varepsilon^{1 / 3}\right)$. So meas $\Omega>0$ when $\varepsilon$ is small enough. Therefore, when $\omega \in \Omega$, by the definition of $F_{k, r j d l}$, from Lemmas 3.3 and 3.4 , for $|k| \geq 1$,

$$
\left|F_{k, r j d l}\right| \leq \frac{|k|^{m+3}}{16 \varrho \varepsilon^{1 / 3}}\left|g_{k, r j d l}\right| .
$$

It follows from 3.10 that

$$
\left|F_{k, r j d l}\right| \leq C \frac{|k|^{m+3}}{\varepsilon^{1 / 3}\left(\lambda_{r} \lambda_{j} \lambda_{d} \lambda_{l}\right)^{1 / 4}}\left|G_{k,|r||j||d||l|}\right|,
$$

which together with $3.20,3.21$ and 3.22 yield that

$$
\left|F_{k, r j d l}\right| \leq C \frac{|k|^{m+3}}{\varepsilon^{1 / 3}\left(\lambda_{r} \lambda_{j} \lambda_{d} \lambda_{l}\right)^{1 / 4}} \sum_{\tau, \tau \pm|r| \pm|j| \pm|d| \pm|l|=0}\left|g_{k}^{\tau}\right|,
$$

where $C$ is dependent on $\varrho$ and $\mu$. In addition, supposing that

$$
F_{0 r}=\left|\sum_{\substack{j, d, l \\|r| \pm|j| \pm|d| \pm|l|=0}}^{\prime} F_{r j d l} w_{j} w_{d} w_{l}\right| \text { and } F_{1 r}=\left|\sum_{|k| \geq 1} \sum_{j, d, l}^{\prime} F_{k, r j d l} e^{\mathrm{i}\langle k, \vartheta\rangle} w_{j} w_{d} w_{l}\right| \text {, }
$$

it is clear that

$$
\left|\frac{\partial F}{\partial w_{r}}\right|^{2} \leq C\left(F_{0 r}\right)^{2}+C\left(F_{1 r}\right)^{2} .
$$

Now we calculate $\left(F_{0 r}\right)^{2}$ and $\left(F_{1 r}\right)^{2}$, respectively.

First, we estimate $\left(F_{0 r}\right)^{2}$. From (3.18),

$$
F_{0 r} \leq C \sum_{\substack{j, d, l \\|r| \pm|j| \pm|d| \pm|l|=0}}^{\prime} \frac{\left|w_{j} w_{d} w_{l}\right|}{\left(\lambda_{r} \lambda_{j} \lambda_{d} \lambda_{l}\right)^{1 / 4}}
$$

When $r=0$ or -0 , we have that

$$
\begin{aligned}
F_{00}, F_{0-0} \leq & \frac{C}{\lambda_{0}^{1 / 4}} \sum_{\substack{j, d, l \\
\pm|j| \pm|d| \pm|l|=0}}^{\prime} \frac{\left|w_{j} w_{d} w_{l}\right|}{\left(\lambda_{j} \lambda_{d} \lambda_{l}\right)^{1 / 4}} \\
\leq & \frac{C}{\lambda_{0}^{1 / 4}}\left[\frac{\left|w_{0}\right|^{3}+\left|w_{-0}\right|^{3}+3\left|w_{0}^{2} w_{-0}\right|+3\left|w_{0} w_{-0}^{2}\right|}{\lambda_{0}^{3 / 4}}+3\left(\frac{\left|w_{0}\right|+\left|w_{-0}\right|}{\lambda_{0}^{1 / 4}}\right)\right. \\
& \left.\quad \sum_{\substack{j, d \neq 0 \text { and }-0 \\
\pm|j| \pm|d|=0}}^{\prime} \frac{\left|w_{j} w_{d}\right|}{\left(\lambda_{j} \lambda_{d}\right)^{1 / 4}}+\sum_{\substack{j, d, l \neq 0 \text { and }-0 \\
\pm|j| \pm|d| \pm|l|=0}}^{\prime} \frac{\left|w_{j} w_{d} w_{l}\right|}{\left(\lambda_{j} \lambda_{d} \lambda_{l}\right)^{1 / 4}}\right]
\end{aligned}
$$




$$
\begin{aligned}
\leq \frac{C}{\lambda_{0}^{1 / 4}} & {\left[\left(\frac{\left|w_{0}\right|+\left|w_{-0}\right|}{\lambda_{0}^{1 / 4}}\right)^{3}+\left(\frac{\left|w_{0}\right|+\left|w_{-0}\right|}{\lambda_{0}^{1 / 4}}\right) \sum_{\substack{j, d \neq 0 \text { and }-0 \\
\pm|j| \pm|d|=0}}^{\prime} \frac{\left|w_{j} w_{d}\right|}{\left(\lambda_{j} \lambda_{d}\right)^{1 / 4}}\right.} \\
& \left.+\sum_{\substack{j, d, l \neq 0 \text { and }-0 \\
\pm|j| \pm|d| \pm|l|=0}}^{\prime} \frac{\left|w_{j} w_{d} w_{l}\right|}{\left(\lambda_{j} \lambda_{d} \lambda_{l}\right)^{1 / 4}}\right] .
\end{aligned}
$$

So

$$
\begin{aligned}
F_{00}^{2}, F_{0-0}^{2} \leq \frac{C}{\lambda_{0}^{1 / 2}} & {\left[\left(\frac{\left|w_{0}\right|+\left|w_{-0}\right|}{\lambda_{0}^{1 / 4}}\right)^{6}+\left(\frac{\left|w_{0}\right|+\left|w_{-0}\right|}{\lambda_{0}^{1 / 4}}\right)^{2}\left(\sum_{\substack{j, d \neq 0 \text { and }-0 \\
\pm|j| \pm|d|=0}}^{\prime} \frac{\left|w_{j} w_{d}\right|}{\left(\lambda_{j} \lambda_{d}\right)^{1 / 4}}\right)^{2}\right.} \\
& \left.+\left(\sum_{\substack{j, d, l \neq 0 \text { and }-0 \\
\pm|j| \pm|d| \pm|l|=0}}^{\prime} \frac{\left|w_{j} w_{d} w_{l}\right|}{\left(\lambda_{j} \lambda_{d} \lambda_{l}\right)^{1 / 4}}\right)^{2}\right] .
\end{aligned}
$$

Assume

$$
\widetilde{w}_{j}:=\frac{\left|w_{j}\right|+\left|w_{-j}\right|}{\lambda_{j}^{1 / 4}}, \quad j \in \mathbb{Z} .
$$

Clearly, $\widetilde{\omega} \in l_{b}^{a, s+1 / 2}$. It follows that

$$
\begin{aligned}
F_{00}^{2}, F_{0-0}^{2} & \leq \frac{C}{\lambda_{0}^{1 / 2}}\left[\widetilde{w}_{0}^{6}+\widetilde{w}_{0}^{2}\left(\sum_{j, d \neq 0, j+d=0} \widetilde{w}_{j} \widetilde{w}_{d}\right)^{2}+\left(\sum_{j, d, l \neq 0, j+d+l=0} \widetilde{w}_{j} \widetilde{w}_{d} \widetilde{w}_{l}\right)^{2}\right] \\
& =\frac{C}{\lambda_{0}^{1 / 2}}\left[\widetilde{w}_{0}^{6}+\widetilde{w}_{0}^{2}(\widetilde{w} * \widetilde{w})_{0}^{2}+(\widetilde{w} * \widetilde{w} * \widetilde{w})_{0}^{2}\right] \\
& =\frac{C}{\lambda_{0}^{1 / 2}}\left[\left|\widetilde{w}_{0}\right|^{6}+\left|\widetilde{w}_{0}\right|^{2}\left|(\widetilde{w} * \widetilde{w})_{0}\right|^{2}+\left|(\widetilde{w} * \widetilde{w} * \widetilde{w})_{0}\right|^{2}\right] .
\end{aligned}
$$

When $r \neq 0$ and -0 , we have that

$$
\begin{aligned}
F_{0 r} \leq \frac{C}{\lambda_{r}^{1 / 4}} & {\left[\left(\frac{\left|w_{0}\right|^{2}+\left|w_{-0}\right|^{2}+2\left|w_{0} w_{-0}\right|}{\lambda_{0}^{1 / 2}}\right) \sum_{\substack{j \neq 0 \text { and }-0 \\
|r| \pm|j|=0}}^{\prime} \frac{\left|w_{j}\right|}{\lambda_{j}^{1 / 4}}\right.} \\
& +\left(\frac{\left|w_{0}\right|+\left|w_{-0}\right|}{\lambda_{0}^{1 / 4}}\right) \sum_{\substack{j, d \neq 0 \text { and }-0 \\
|r| \pm|j| \pm|d|=0}}^{\prime} \frac{\left|w_{j} w_{d}\right|}{\left(\lambda_{j} \lambda_{d}\right)^{1 / 4}} \\
& \left.+\sum_{\substack{j, d, l \neq 0 \text { and }-0 \\
|r| \pm|j| \pm|d| \pm|l|=0}}^{\prime} \frac{\left|w_{j} w_{d} w_{l}\right|}{\left(\lambda_{j} \lambda_{d} \lambda_{l}\right)^{1 / 4}}\right] .
\end{aligned}
$$


So, by using of 3.25,

$$
\begin{aligned}
& F_{0 r}^{2} \leq \frac{C}{\lambda_{r}^{1 / 2}} {\left[\left(\frac{\left|w_{0}\right|+\left|w_{-0}\right|}{\lambda_{0}^{1 / 4}}\right)^{4}\left(\sum_{\substack{j \neq 0 \text { and }-0 \\
|r| \pm|j|=0}}^{\prime} \frac{\left|w_{j}\right|}{\lambda_{j}^{1 / 4}}\right)^{2}\right.} \\
&+\left(\frac{\left|w_{0}\right|+\left|w_{-0}\right|}{\lambda_{0}^{1 / 4}}\right)^{2}\left(\sum_{\substack{j, d \neq 0 \text { and }-0 \\
|r| \pm|j| \pm|d|=0}}^{\prime} \frac{\left|w_{j} w_{d}\right|}{\left(\lambda_{j} \lambda_{d}\right)^{1 / 4}}\right)^{2} \\
&\left.+\left(\sum_{\substack{j, d, l \neq 0 \text { and }-0 \\
|r| \pm|j| \pm|d| \pm|l|=0}}^{\prime} \frac{\left|w_{j} w_{d} w_{l}\right|}{\left(\lambda_{j} \lambda_{d} \lambda_{l}\right)^{1 / 4}}\right)^{2}\right] \\
& \leq \frac{C}{\lambda_{r}^{1 / 2}}\left[\left|\widetilde{w}_{0}\right|^{4}\left|\widetilde{w}_{r}\right|^{2}+\left|\widetilde{w}_{0}\right|^{2}\left|(\widetilde{w} * \widetilde{w})_{r}\right|^{2}+\left|(\widetilde{w} * \widetilde{w} * \widetilde{w})_{r}\right|^{2}\right] .
\end{aligned}
$$

Second, we estimate $F_{1 r}^{2}$. By using of Lemma 3.4 .

$$
\begin{aligned}
F_{1 r}^{2} & \leq C \sum_{|k| \geq 1}[k]^{2 m+1}\left|\sum_{j, d, l}^{\prime} F_{k, r j d l} e^{\mathrm{i}\langle k, \vartheta\rangle} w_{j} w_{d} w_{l}\right|^{2} \\
& \leq C \sum_{|k| \geq 1}[k]^{2 m+1}\left(\sum_{j, d, l}^{\prime}\left|F_{k, r j d l}\right|\left|e^{\mathrm{i}\langle k, \vartheta\rangle}\right|\left|w_{j} w_{d} w_{l}\right|\right)^{2} \\
& =C \sum_{|k| \geq 1}[k]^{2 m+1}\left|e^{\mathrm{i}\langle k, \vartheta\rangle}\right|^{2}\left(\sum_{j, d, l}^{\prime}\left|F_{k, r j d l}\right|\left|w_{j} w_{d} w_{l}\right|\right)^{2} .
\end{aligned}
$$

It follows from 3.23 that

$$
F_{1 r}^{2} \leq \frac{C}{\varepsilon^{2 / 3}\left(\lambda_{r}\right)^{1 / 2}} \sum_{|k| \geq 1}[k]^{4 m+7}\left|e^{\mathrm{i}\langle k, \vartheta\rangle}\right|^{2}\left(\sum_{j, d, l}^{\prime} \sum_{\tau, \tau \pm|r| \pm|j| \pm|d| \pm|l|=0}\left|g_{k}^{\tau}\right| \frac{\left|w_{j} w_{d} w_{l}\right|}{\left(\lambda_{j} \lambda_{d} \lambda_{l}\right)^{1 / 4}}\right)^{2}
$$

However, when $r=0$ or -0 ,

$$
\begin{aligned}
& \sum_{j, d, l}^{\prime} \sum_{\tau, \tau \pm|r| \pm|j| \pm|d| \pm|l|=0}\left|g_{k}^{\tau}\right| \frac{\left|w_{j} w_{d} w_{l}\right|}{\left(\lambda_{j} \lambda_{d} \lambda_{l}\right)^{1 / 4}} \\
& =\left(\frac{\left|w_{0}\right|+\left|w_{-0}\right|}{\lambda_{0}^{1 / 4}}\right)^{3}\left|g_{k}^{0}\right|+\left(\frac{\left|w_{0}\right|+\left|w_{-0}\right|}{\lambda_{0}^{1 / 4}}\right)^{2} \sum_{j \neq 0 \text { and }-0}^{\prime} \sum_{\tau, \tau \pm|j|=0}\left|g_{k}^{\tau}\right| \frac{\left|w_{j}\right|}{\lambda_{j}^{1 / 4}} \\
& +\left(\frac{\left|w_{0}\right|+\left|w_{-0}\right|}{\lambda_{0}^{1 / 4}}\right)_{j, d \neq 0 \text { and }-0} \sum_{\tau, \tau \pm|j| \pm|d|=0}^{\prime}\left|g_{k}^{\tau}\right| \frac{\left|w_{j} w_{d}\right|}{\left(\lambda_{j} \lambda_{d}\right)^{1 / 4}} \\
& +\sum_{j, d, l \neq 0 \text { and }-0}^{\prime} \sum_{\tau, \tau \pm|j| \pm|d| \pm|l|=0}\left|g_{k}^{\tau}\right| \frac{\left|w_{j} w_{d} w_{l}\right|}{\left(\lambda_{j} \lambda_{d} \lambda_{l}\right)^{1 / 4}}
\end{aligned}
$$


holds. Thus, it follows from 3.28 that

$$
\begin{aligned}
F_{10}^{2}, F_{1-0}^{2} \leq & \frac{C}{\varepsilon^{2 / 3} \lambda_{0}^{1 / 2}} \sum_{|k| \geq 1}[k]^{4 m+7}\left|e^{\mathrm{i}\langle k, \vartheta\rangle}\right|^{2} \\
& \times\left[\left(\frac{\left|w_{0}\right|+\left|w_{-0}\right|}{\lambda_{0}^{1 / 4}}\right)^{6}\left|g_{k}^{0}\right|^{2}+\left(\frac{\left|w_{0}\right|+\left|w_{-0}\right|}{\lambda_{0}^{1 / 4}}\right)^{4}\left(\sum_{j \neq 0 \text { and }-0}^{\prime} \sum_{\tau, \tau \pm|j|=0}\left|g_{k}^{\tau}\right| \frac{\left|w_{j}\right|}{\lambda_{j}^{1 / 4}}\right)^{2}\right. \\
& +\left(\frac{\left|w_{0}\right|+\left|w_{-0}\right|}{\lambda_{0}}\right)^{2}\left(\sum_{j, d \neq 0 \text { and }-0}^{\prime} \sum_{\tau, \tau \pm|j| \pm|d|=0}\left|g_{k}^{\tau}\right| \frac{\left|w_{j} w_{d}\right|}{\left(\lambda_{j} \lambda_{d}\right)^{1 / 4}}\right)^{2} \\
& \left.+\left(\sum_{j, d, l \neq 0 \text { and }-0}^{\prime} \sum_{\tau, \tau \pm|j| \pm|d| \pm|l|=0}\left|g_{k}^{\tau}\right| \frac{\left|w_{j} w_{d} w_{l}\right|}{\left(\lambda_{j} \lambda_{d} \lambda_{l}\right)^{1 / 4}}\right)^{2}\right] .
\end{aligned}
$$

We define $\widetilde{g}_{k}^{\tau}:=\left|g_{k}^{\tau}\right|+\left|g_{k}^{-\tau}\right|$ for $0 \neq \tau \in \mathbb{Z}, \widetilde{g}_{k}^{0}:=2\left|g_{k}^{0}\right|$, and

$$
\widetilde{g}_{k}:=\left\{\ldots, \widetilde{g}_{k}^{2}, \widetilde{g}_{k}^{-1}, \widetilde{g}_{k}^{0}, \widetilde{g}_{k}^{1}, \widetilde{g}_{k}^{2}, \ldots\right\}
$$

for every $0 \neq k \in \mathbb{Z}^{m}$. For every $0 \neq k \in \mathbb{Z}^{m}$, by Lemma A.1 in $\left[12\right.$, from $\left(\mathrm{H}_{1}\right),\left(\mathrm{H}_{2}\right)$ and (3.19,

$$
\left|g_{k}^{r}\right|<|g(\vartheta, x)|_{D_{1}\left(\sigma_{1}\right) \times D_{2}(2 a)} e^{-|k| \sigma_{1}} e^{-2 a|r|}
$$

is always true for $r \in \mathbb{Z}$. Hence, for all $(\vartheta, x) \in D_{1}\left(\sigma_{1}\right) \times D_{2}(2 a)$,

$$
\begin{aligned}
\left(\left\|\widetilde{g}_{k}\right\|_{a, s+1 / 2}^{1}\right)^{2} & =\left(\widetilde{g}_{k}^{0}\right)^{2}+\sum_{|r| \geq 1}\left(\widetilde{g}_{k}^{r}\right)^{2} e^{2 a|r|}|r|^{2 s+1} \\
& \leq\left(|g(\vartheta, x)|_{D_{1}\left(\sigma_{1}\right) \times D_{2}(2 a)}\right)^{2} e^{-2|k| \sigma_{1}}\left(1+\sum_{|r| \geq 1} e^{-2 a|r|}|r|^{2 s+1}\right)
\end{aligned}
$$

Since the series $\sum_{|r| \geq 1} e^{-2 a|r|}|r|^{2 s+1}$ is convergent, the above inequality is bounded and

$$
\left(\left\|\widetilde{g}_{k}\right\|_{a, s+1 / 2}^{1}\right)^{2} \leq C e^{-2|k| \sigma_{1}}
$$

holds, where the constant $C$ depends on $g, \sigma_{1}, a$ and $s$. Thus, for fixed $k \in \mathbb{Z}, \widetilde{g}_{k} \in l_{b}^{a, s+1 / 2}$. It derives from 3.29 and 3.25 that

$$
\begin{aligned}
& F_{10}^{2}, F_{1-0}^{2} \leq \frac{C}{\varepsilon^{2 / 3} \lambda_{0}^{1 / 2}} \sum_{|k| \geq 1}[k]^{4 m+7}\left|e^{\mathrm{i}\langle k, \vartheta\rangle}\right|^{2}\left[\left|\widetilde{w}_{0}\right|^{6}\left|\widetilde{g}_{k}^{0}\right|^{2}+\left|\widetilde{w}_{0}\right|^{4}\left|\left(\widetilde{g}_{k} * \widetilde{w}\right)_{0}\right|^{2}\right. \\
& \left.+\left|\widetilde{w}_{0}\right|^{2}\left|\left(\widetilde{g}_{k} * \widetilde{w} * \widetilde{w}\right)_{0}\right|^{2}+\left|\left(\widetilde{g}_{k} * \widetilde{w} * \widetilde{w} * \widetilde{w}\right)_{0}\right|^{2}\right] .
\end{aligned}
$$


When $r \neq 0$ and -0 ,

$$
\begin{aligned}
& \sum_{j, d, l}^{\prime} \sum_{\tau, \tau \pm|r| \pm|j| \pm|d| \pm|l|=0}\left|g_{k}^{\tau}\right| \frac{\left|w_{j} w_{d} w_{l}\right|}{\left(\lambda_{j} \lambda_{d} \lambda_{l}\right)^{1 / 4}} \\
& =\left(\frac{\left|w_{0}\right|+\left|w_{-0}\right|}{\lambda_{0}^{1 / 4}}\right)^{3} \sum_{\tau \pm|r|=0}^{\prime}\left|g_{k}^{\tau}\right|+\left(\frac{\left|w_{0}\right|+\left|w_{-0}\right|}{\lambda_{0}^{1 / 4}}\right)^{2} \sum_{j \neq 0 \text { and }-0}^{\prime} \sum_{\tau, \tau \pm|r| \pm|j|=0}\left|g_{k}^{\tau}\right| \frac{\left|w_{j}\right|}{\lambda_{j}^{1 / 4}} \\
& +\left(\frac{\left|w_{0}\right|+\left|w_{-0}\right|}{\lambda_{0}^{1 / 4}}\right)_{j, d \neq 0 \text { and }-0} \sum_{\tau, \tau \pm|r| \pm|j| \pm|d|=0}^{\prime}\left|g_{k}^{\tau}\right| \frac{\left|w_{j} w_{d}\right|}{\left(\lambda_{j} \lambda_{d}\right)^{1 / 4}} \\
& +\sum_{j, d, l \neq 0 \text { and }-0 \tau, \tau \pm|r| \pm|j| \pm|d| \pm|l|=0}^{\prime}\left|g_{k}^{\tau}\right| \frac{\left|w_{j} w_{d} w_{l}\right|}{\left(\lambda_{j} \lambda_{d} \lambda_{l}\right)^{1 / 4}} .
\end{aligned}
$$

It follows from (3.28) and 3.25 that

$$
\begin{aligned}
& F_{1 r}^{2} \leq \frac{C}{\varepsilon^{2 / 3} \lambda_{r}^{1 / 2}} \sum_{|k| \geq 1}[k]^{4 m+7}\left|e^{\mathrm{i}\langle k, \vartheta\rangle}\right|^{2}\left[\left(\frac{\left|w_{0}\right|+\left|w_{-0}\right|}{\lambda_{0}^{1 / 4}}\right)^{6}\left(\sum_{\tau \pm|r|=0}^{\prime}\left|g_{k}^{\tau}\right|\right)^{2}\right. \\
& +\left(\frac{\left|w_{0}\right|+\left|w_{-0}\right|}{\lambda_{0}^{1 / 4}}\right)^{4}\left(\sum_{j \neq 0 \text { and }-0}^{\prime} \sum_{\tau, \tau \pm|r| \pm|j|=0}\left|g_{k}^{\tau}\right| \frac{\left|w_{j}\right|}{\left(\lambda_{j}\right)^{1 / 4}}\right)^{2} \\
& +\left(\frac{\left|w_{0}\right|+\left|w_{-0}\right|}{\lambda_{0}^{1 / 4}}\right)^{2}\left(\sum_{j, d \neq 0 \text { and }-0}^{\prime} \sum_{\tau, \tau \pm|r| \pm|j| \pm|d|=0}\left|g_{k}^{\tau}\right| \frac{\left|w_{j} w_{d}\right|}{\left(\lambda_{j} \lambda_{d}\right)^{1 / 4}}\right)^{2} \\
& \left.+\left(\sum_{j, d, l \neq 0 \text { and }-0}^{\prime} \sum_{\tau, \tau \pm|r| \pm|j| \pm|d| \pm|l|=0}\left|g_{k}^{\tau}\right| \frac{\left|w_{j} w_{d} w_{l}\right|}{\left(\lambda_{j} \lambda_{d} \lambda_{l}\right)^{1 / 4}}\right)^{2}\right] \\
& \leq \frac{C}{\lambda_{r}^{1 / 2} \varepsilon^{2 / 3}} \sum_{|k| \geq 1}[k]^{4 m+7}\left|e^{\mathrm{i}\langle k, \vartheta\rangle}\right|^{2}\left(\left|\widetilde{w}_{0}\right|^{6}\left|\widetilde{g}_{k}^{r}\right|^{2}+\left|\widetilde{w}_{0}\right|^{4}\left|\left(\widetilde{g}_{k} * \widetilde{w}\right)_{r}\right|^{2}\right. \\
& \left.+\left|\widetilde{w}_{0}\right|^{2}\left|\left(\widetilde{g}_{k} * \widetilde{w} * \widetilde{w}\right)_{r}\right|^{2}+\left|\left(\widetilde{g}_{k} * \widetilde{w} * \widetilde{w} * \widetilde{w}\right)_{r}\right|^{2}\right) .
\end{aligned}
$$

Third, we calculate $\left(\left\|F_{w}\right\|_{a, s+1}\right)^{2}$. On one hand, from (3.26) and (3.27), we obtain that

$$
\begin{aligned}
& F_{00}^{2}+F_{0-0}^{2}+\sum_{|r| \geq 1} F_{0 r}^{2}|r|^{2 s+2} e^{2 a|r|} \\
\leq C & {\left[\left|\widetilde{w}_{0}\right|^{6}+\left|\widetilde{w}_{0}\right|^{2}\left|(\widetilde{w} * \widetilde{w})_{0}\right|^{2}+\left|(\widetilde{w} * \widetilde{w} * \widetilde{w})_{0}\right|^{2}+\left|\widetilde{w}_{0}\right|^{4} \sum_{|r| \geq 1}\left|\widetilde{w}_{r}\right|^{2}|r|^{2 s+1} e^{2 a|r|}\right.} \\
& \left.+\left|\widetilde{w}_{0}\right|^{2} \sum_{|r| \geq 1}\left|(\widetilde{w} * \widetilde{w})_{r}\right|^{2}|r|^{2 s+1} e^{2 a|r|}+\sum_{|r| \geq 1}\left|(\widetilde{w} * \widetilde{w} * \widetilde{w})_{r}\right|^{2}|r|^{2 s+1} e^{2 a|r|}\right] \\
=C & {\left[\left|\widetilde{w}_{0}\right|^{4}\left(\left|\widetilde{w}_{0}\right|^{2}+\sum_{|r| \geq 1}\left|\widetilde{w}_{r}\right|^{2}|r|^{2 s+1} e^{2 a|r|}\right)\right.} \\
& +\left|\widetilde{w}_{0}\right|^{2}\left(\left|(\widetilde{w} * \widetilde{w})_{0}\right|^{2}+\sum_{|r| \geq 1}\left|(\widetilde{w} * \widetilde{w})_{r}\right|^{2}|r|^{2 s+1} e^{2 a|r|}\right)
\end{aligned}
$$




$$
\begin{aligned}
&\left.+\left(\left|(\widetilde{w} * \widetilde{w} * \widetilde{w})_{0}\right|^{2}+\sum_{|r| \geq 1}\left|(\widetilde{w} * \widetilde{w} * \widetilde{w})_{r}\right|^{2}|r|^{2 s+1} e^{2 a|r|}\right)\right] \\
&=C\left[\left|\widetilde{w}_{0}\right|^{4}\left(\|\widetilde{w}\|_{a, s+1 / 2}^{1}\right)^{2}+\left|\widetilde{w}_{0}\right|^{2}\left(\|\widetilde{w} * \widetilde{w}\|_{a, s+1 / 2}^{1}\right)^{2}+\left(\|\widetilde{w} * \widetilde{w} * \widetilde{w}\|_{a, s+1 / 2}^{1}\right)^{2}\right]
\end{aligned}
$$

holds. By using of Lemma 2.8, it follows that

$$
\begin{aligned}
& F_{00}^{2}+F_{0-0}^{2}+\sum_{|r| \geq 1} F_{0 r}^{2}|r|^{2 s+2} e^{2 a|r|} \\
\leq & C\left[\left|\widetilde{w}_{0}\right|^{4}\left(\|\widetilde{w}\|_{a, s+1 / 2}^{1}\right)^{2}+\left|\widetilde{w}_{0}\right|^{2}\left(\|\widetilde{w}\|_{a, s+1 / 2}^{1}\right)^{4}+\left(\|\widetilde{w}\|_{a, s+1 / 2}^{1}\right)^{6}\right] \\
\leq & C\left(\|\widetilde{w}\|_{a, s+1 / 2}^{1}\right)^{6} \leq C\left(\|w\|_{a, s}^{2}\right)^{6} .
\end{aligned}
$$

On the other hand, from (3.31) and 3.32 , we obtain that

$$
\begin{aligned}
& F_{10}^{2}+F_{1-0}^{2}+\sum_{|r| \geq 1} F_{1 r}^{2}|r|^{2 s+2} e^{2 a|r|} \\
& \leq \frac{C}{\varepsilon^{2 / 3}} \sum_{|k| \geq 1}[k]^{4 m+7}\left|e^{\mathrm{i}\langle k, \vartheta\rangle}\right|^{2}\left[\left|\widetilde{w}_{0}\right|^{6}\left(\left|\widetilde{g}_{k}^{0}\right|^{2}+\sum_{|r| \geq 1}\left|\widetilde{g}_{k}^{r}\right|^{2} e^{2 a|r|}|r|^{2 s+1}\right)\right. \\
& +\left|\widetilde{w}_{0}\right|^{4}\left(\left|\left(\widetilde{g}_{k} * \widetilde{w}\right)_{0}\right|^{2}+\sum_{|r| \geq 1}\left|\left(\widetilde{g}_{k} * \widetilde{w}\right)_{r}\right|^{2} e^{2 a|r|}|r|^{2 s+1}\right) \\
& \quad+\left|\widetilde{w}_{0}\right|^{2}\left(\left|\left(\widetilde{g}_{k} * \widetilde{w} * \widetilde{w}\right)_{0}\right|^{2}+\sum_{|r| \geq 1}\left|\left(\widetilde{g}_{k} * \widetilde{w} * \widetilde{w}\right)_{r}\right|^{2} e^{2 a|r|}|r|^{2 s+1}\right) \\
& \left.\quad+\left(\left|\left(\widetilde{g}_{k} * \widetilde{w} * \widetilde{w} * \widetilde{w}\right)_{0}\right|^{2}+\sum_{|r| \geq 1}\left|\left(\widetilde{g}_{k} * \widetilde{w} * \widetilde{w} * \widetilde{w}\right)_{r}\right|^{2} e^{2 a|r|}|r|^{2 s+1}\right)\right] \\
& \leq \frac{C}{\varepsilon^{2 / 3}} \sum_{|k| \geq 1}[k]^{4 m+7}\left|e^{\mathrm{i}\langle k, \vartheta\rangle}\right|^{2}\left[\left|\widetilde{w}_{0}\right|^{6}\left(\left\|\widetilde{g}_{k}\right\|_{a, s+1 / 2}^{1}\right)^{2}+\left|\widetilde{w}_{0}\right|^{4}\left(\left\|\widetilde{g}_{k} * \widetilde{w}\right\|_{a, s+1 / 2}^{1}\right)^{2}\right. \\
& \left.\quad+\left|\widetilde{w}_{0}\right|^{2}\left(\left\|\widetilde{g}_{k} * \widetilde{w} * \widetilde{w}\right\|_{a, s+1 / 2}^{1}\right)^{2}+\left(\left\|\widetilde{g}_{k} * \widetilde{w} * \widetilde{w} * \widetilde{w}\right\|_{a, s+1 / 2}^{1}\right)^{2}\right] .
\end{aligned}
$$

By using of Lemma 2.8, we get

$$
\begin{aligned}
& F_{10}^{2}+F_{1-0}^{2}+\sum_{|r| \geq 1} F_{1 r}^{2}|r|^{2 s+2} e^{2 a|r|} \\
& \leq \frac{C}{\varepsilon^{2 / 3}} \sum_{|k| \geq 1}[k]^{4 m+7}\left|e^{\mathrm{i}\langle k, \vartheta\rangle}\right|^{2}\left[\left|\widetilde{w}_{0}\right|^{6}\left(\left\|\widetilde{g}_{k}\right\|_{a, s+1 / 2}^{1}\right)^{2}+\left|\widetilde{w}_{0}\right|^{4}\left(\left\|\widetilde{g}_{k}\right\|_{a, s+1 / 2}^{1}\|\widetilde{w}\|_{a, s+1 / 2}^{1}\right)^{2}\right. \\
& \quad+\left|\widetilde{w}_{0}\right|^{2}\left(\left\|\widetilde{g}_{k}\right\|_{a, s+1 / 2}^{1}\|\widetilde{w}\|_{a, s+1 / 2}^{1}\|\widetilde{w}\|_{a, s+1 / 2}^{1}\right)^{2} \\
& \left.\quad+\left(\left\|\widetilde{g}_{k}\right\|_{a, s+1 / 2}^{1}\|\widetilde{w}\|_{a, s+1 / 2}^{1}\|\widetilde{w}\|_{a, s+1 / 2}^{1}\|\widetilde{w}\|_{a, s+1 / 2}^{1}\right)^{2}\right] .
\end{aligned}
$$

It follows from 3.30 that, for all $(\vartheta, x) \in D_{1}\left(\sigma_{1} / 2\right) \times D_{2}(2 a)$,

$$
F_{10}^{2}+F_{1-0}^{2}+\sum_{|r| \geq 1} F_{1 r}^{2}|r|^{2 s+2} e^{2 a|r|} \leq \frac{C}{\varepsilon^{2 / 3}}\left(\|\widetilde{w}\|_{a, s+1 / 2}^{1}\right)^{6}\left(\sum_{|k| \geq 1}[k]^{4 m+7} e^{|k| \sigma_{1}} \cdot e^{-2|k| \sigma_{1}}\right)
$$


However, it is well known that the number

$$
\sharp\left\{k \in \mathbb{Z}^{m}:|k|=l\right\} \leq 2^{m} l^{m-1}, \quad l \in \mathbb{Z}^{+}:=\{1,2, \ldots\} .
$$

Thus, by using of the convergence of the series $\sum_{1 \leq|k|=l}^{\infty} 2^{m} l^{5 m+6} e^{-l \sigma_{1}}$,

$$
\sum_{|k| \geq 1}[k]^{4 m+7} e^{|k| \sigma_{1}} \cdot e^{-2|k| \sigma_{1}}<\sum_{1 \leq|k|=l}^{\infty} 2^{m} l^{m-1} l^{4 m+7} e^{-l \sigma_{1}} \leq C,
$$

where $C$ is a bounded constant depending only on $\sigma_{1}$ and $m$. It derives from (3.34) that

$$
F_{10}^{2}+F_{1-0}^{2}+\sum_{|r| \geq 1} F_{1 r}^{2}|r|^{2 s+2} e^{2 a|r|} \leq \frac{C}{\varepsilon^{2 / 3}}\left(\|\widetilde{w}\|_{a, s+1 / 2}^{1}\right)^{6} \leq \frac{C}{\varepsilon^{2 / 3}}\left(\|w\|_{a, s}^{2}\right)^{6},
$$

where the constant $C$ depends on $\varrho, \mu, g, \sigma_{1}, n, a, m$ and $s$. By using of (3.24),

$$
\begin{aligned}
\left(\left\|\frac{\partial F}{\partial w}\right\|_{a, s+1}^{2}\right)^{2}= & \left|\frac{\partial F}{\partial w_{0}}\right|^{2}+\left|\frac{\partial F}{\partial w_{-0}}\right|^{2}+\sum_{|r| \geq 1}\left|\frac{\partial F}{\partial w_{r}}\right|^{2} e^{2 a|r|}|r|^{2 s+2} \\
\leq & C\left(F_{00}^{2}+F_{10}^{2}+F_{0-0}^{2}+F_{1-0}^{2}+\sum_{|r| \geq 1}\left(F_{0 r}^{2}+F_{1 r}^{2}\right)|r|^{2 s+2} e^{2 a|r|}\right) \\
= & C\left(F_{00}^{2}+F_{0-0}^{2}+\sum_{|r| \geq 1}\left(F_{0 r}^{2}\right)|r|^{2 s+2} e^{2 a|r|}\right) \\
& +C\left(F_{10}^{2}+F_{1-0}^{2}+\sum_{|r| \geq 1}\left(F_{1 r}^{2}\right)|r|^{2 s+2} e^{2 a|r|}\right)
\end{aligned}
$$

holds true. It follows from (3.33) and (3.36) that $\left(\left\|\frac{\partial F}{\partial w}\right\|_{a, s+1}^{2}\right)^{2} \leq \frac{C}{\varepsilon^{2 / 3}}\left(\|w\|_{a, s}^{2}\right)^{6}$, where the constant $C$ depends on $\varrho, n, \mu, g, \sigma_{1}, a, m$ and $s$. Therefore, (3.17) is true. The analyticity of $F_{w}$ follows from the analyticity of each component function and its local boundedness. This completes proving the Claim.

Similarly, we can prove that, for all $(\vartheta, x) \in D_{1}\left(\sigma_{1} / 2\right) \times D_{2}(2 a),|\check{G}| \leq C\left(\|Z\|_{a, s}\right)^{4}$, where $C$ depends on $\mu, g, \sigma_{1}, a$ and $s$.

Step 3. From (3.17), it follows that the time-1-map $X_{F}^{1}$ is well defined in a sufficiently small neighborhood of the origin in $l^{a, s}$ with the estimates

$$
\|\Psi-\mathrm{id}\|_{a, s+1}=\mathcal{O}\left(\|w\|_{a, s}^{3}\right), \quad\|D \Psi-\mathrm{Id}\|_{a, s+1, s}^{\mathrm{op}}=\mathcal{O}\left(\|w\|_{a, s}^{2}\right),
$$

with the operator norm $\|A\|_{a, r, s}^{\text {op }}=\sup _{w \neq 0} \frac{\|A w\|_{a, r}}{\|w\|_{a, s}}$. Since

$$
\|D \Psi-\operatorname{Id}\|_{a, s+1, s+1}^{\mathrm{op}} \leq\|D \Psi-\mathrm{Id}\|_{a, s+1, s}^{\mathrm{op}},
$$

$D \Psi$ defines an isomorphism of $l^{a, s+1}$ in a sufficiently small neighborhood of the origin. It follows that with $X_{H} \in \mathcal{A}\left(l^{a, s}, l^{a, s+1}\right)$, also

$$
\Psi^{*} X_{H}=D \Psi^{-1} X_{H} \circ \Psi=X_{H \circ \Psi} \in \mathcal{A}\left(l^{a, s}, l^{a, s+1}\right) .
$$


The same holds for the Lie bracket: the boundedness of $\left\|D X_{F}\right\|_{a, s+1, s}^{\mathrm{op}}$ implies that

$$
\left[X_{F}, X_{H}\right]=X_{\{H, F\}} \in \mathcal{A}\left(l^{a, s}, l^{a, s+1}\right) .
$$

These two facts show that $X_{K} \in \mathcal{A}\left(l^{a, s}, l^{a, s+1}\right)$. The analogous claims for $X_{\bar{G}}$ and $X_{\breve{G}}$ are obvious. Suppose that

$$
K=\{G, F\}+\int_{0}^{1}(1-t)\{\{H, F\}, F\} \circ X_{\mathcal{F}}^{t} d t .
$$

By using of Lemma 2.9 and (3.17), and from the fact that

$$
|\{\Lambda, F\}|=|\bar{G}+\check{G}-G|=\mathcal{O}\left(\left(\|w\|_{a, s}^{2}\right)^{4}\right),
$$

it results

$$
|\{G, F\}| \leq \frac{C}{\varepsilon^{1 / 3}}\left(\|w\|_{a, s}^{2}\right)^{6} \quad \text { and } \quad|\{\{\Lambda, F\}, F\}| \leq \frac{C}{\varepsilon^{1 / 3}}\left(\|w\|_{a, s}^{2}\right)^{6} .
$$

Moreover,

$$
|\{\{G, F\}, F\}| \leq \frac{C}{\varepsilon^{2 / 3}}\left(\|w\|_{a, s}^{2}\right)^{8}
$$

and the fact that $H=\Lambda+\varepsilon G$ hold. Hence, $|K| \leq \frac{C}{\varepsilon^{1 / 3}}\left(\|w\|_{a, s}^{2}\right)^{6}$, i.e., $\varepsilon^{1 / 3}|K|=\mathcal{O}\left(\|w\|_{a, s}^{2}\right)^{6}$ for $\|w\|_{a, s}^{2} \leq 1$. This completes the proof.

Now let $\mathcal{J}=\{0,1, \ldots, n\}$ first. The case of a more general set $\mathcal{J}$ really makes no difference. We introduce the action-angle variables by setting

$$
z_{j}= \begin{cases}\sqrt{I_{j}} e^{-\mathrm{i} \theta_{j}} & \text { if } 0 \leq j \leq n \\ z_{j} & \text { if } j \geq n+1\end{cases}
$$

The normal form becomes

$$
\Lambda+\varepsilon \bar{G}=\langle\omega, J\rangle+\sum_{0 \leq j \leq n} \sqrt{\lambda_{j}} I_{j}+\sum_{j>n} \sqrt{\lambda_{j}} z_{j} \bar{z}_{j}+\frac{\varepsilon}{2}\langle A I, I\rangle+\varepsilon\langle B I, \widehat{Z}\rangle
$$

with $I=\left(I_{0}, I_{1}, \ldots, I_{n}\right), A=\left(\bar{G}_{i j}\right)_{0 \leq i, j \leq n}, B=\left(\bar{G}_{i j}\right)_{0 \leq j \leq n<i}$ and $\widehat{Z}=\left(\left|z_{n+1}\right|^{2},\left|z_{n+2}\right|^{2}, \ldots\right)$.

Suppose that the parameter vector is $\widetilde{\xi}=\left(\widetilde{\xi}_{j}\right)_{0 \leq j \leq n}$ and the new action variable $\widetilde{\rho}=$ $\left(\widetilde{\rho}_{j}\right)_{0 \leq j \leq n}$ is as follows: $I_{j}=\varepsilon \widetilde{\xi}_{j}+\widetilde{\rho}_{j}, \widetilde{\xi}_{j} \in[0,1],\left|\widetilde{\rho}_{j}\right|<\varepsilon^{2}, 0 \leq j \leq n$, then the normal form is changed into

$$
\begin{aligned}
\Lambda+\varepsilon \bar{G}= & \langle\omega, J\rangle+\sum_{0 \leq j \leq n} \sqrt{\lambda_{j}} \widetilde{\rho}_{j}+\sum_{j>n} \sqrt{\lambda_{j}} z_{j} \bar{z}_{j} \\
& +\frac{\varepsilon^{2}}{2} \sum_{0 \leq i, j \leq n} \bar{G}_{i j} \widetilde{\rho}_{i} \widetilde{\xi}_{j}+\frac{\varepsilon^{2}}{2} \sum_{0 \leq i, j \leq n} \bar{G}_{i j} \widetilde{\xi}_{i} \widetilde{\rho}_{j}+\frac{\varepsilon}{2} \sum_{0 \leq i, j \leq n} \bar{G}_{i j} \widetilde{\rho}_{i} \widetilde{\rho}_{j} \\
& +\varepsilon^{2} \sum_{0 \leq i \leq n<j} \bar{G}_{i j} \widetilde{\xi}_{i}\left|z_{j}\right|^{2}+\varepsilon \sum_{0 \leq j \leq n<i} \bar{G}_{i j} \widetilde{\rho}_{j}\left|z_{i}\right|^{2} .
\end{aligned}
$$


Hence, the total Hamiltonian is

$$
\begin{aligned}
H= & \langle\omega, J\rangle+\sum_{0 \leq j \leq n} \sqrt{\lambda_{j}} \widetilde{\rho}_{j}+\sum_{j>n} \sqrt{\lambda_{j}} z_{j} \bar{z}_{j}+\frac{\varepsilon^{2}}{2} \sum_{0 \leq i, j \leq n} \bar{G}_{i j} \widetilde{\rho}_{i} \widetilde{\xi}_{j} \\
& +\frac{\varepsilon^{2}}{2} \sum_{0 \leq i, j \leq n} \bar{G}_{i j} \widetilde{\xi}_{i} \widetilde{\rho}_{j}+\varepsilon^{2} \sum_{0 \leq j \leq n<i} \bar{G}_{i j} \widetilde{\xi}_{j}\left|z_{i}\right|^{2}+P,
\end{aligned}
$$

where $P=\varepsilon \breve{G}+\varepsilon \check{G}+\varepsilon^{2} K$ and

$$
|\breve{G}|=\mathcal{O}\left(|\widetilde{\rho}|^{2}\right)+\mathcal{O}\left(|\widetilde{\rho}|\|\widehat{Z}\|_{a, s}\right)
$$

At the end of this section, we give the estimates of the perturbed term $P$. At first, we introduce some notations from [10]. Assume that $z=\left(z_{0}, z_{1}, z_{2}, \ldots\right)$ with $\|z\|_{a, s}<\infty$; $l_{n+1}^{a, s}$ is the Hilbert space of all complex sequences $Z=\left\{z_{j} \in \mathbb{C}: j \geq n+1\right\}$ with $\|Z\|_{a, s}:=\|\widetilde{Z}\|_{a, s}, \widetilde{Z}=\left\{\widetilde{z}_{j} \in \mathbb{C}, j \geq 0\right\}, \widetilde{z}_{j}=0$ as $0 \leq j \leq n$ and $\widetilde{z}_{j}=z_{j}$ as $j \geq n+1$; $x^{\prime}=\vartheta \oplus \theta, y=J \oplus \widetilde{\rho}, \zeta=\omega \oplus\left(\widetilde{\xi}_{j}\right)_{0 \leq j \leq n}$, then the phase space is

$$
\mathcal{P}^{a, s}=\widehat{\mathbb{T}}^{m+n+1} \times \mathbb{C}^{m+n+1} \times l_{n+1}^{a, s} \times l_{n+1}^{a, s} \ni\left(x^{\prime}, y, Z, \bar{Z}\right),
$$

and

$$
D(\sigma, r):=\left\{\left(x^{\prime}, y, Z, \bar{Z}\right) \in \mathcal{P}^{a, s}:\left|\operatorname{Im} x^{\prime}\right|<\sigma,|y|<r^{2},\|Z\|_{a, s}+\|\bar{Z}\|_{a, s}<r\right\}
$$

where $\theta=\left(\theta_{j}\right)_{0 \leq j \leq n}, \widehat{\mathbb{T}}^{m+n+1}$ is the complexification of the usual $(m+n+1)$-torus $\mathbb{T}^{m+n+1}$, $\sigma>0$ and $r>0$. We define the weighted phase norms

$$
|W|_{r}=|W|_{\bar{s}, r}=\left|x^{\prime}\right|+\frac{1}{r^{2}}|y|+\frac{1}{r}\|Z\|_{a, \bar{s}}+\frac{1}{r}\|\bar{Z}\|_{a, \bar{s}}
$$

for $W=\left(x^{\prime}, y, Z, \bar{Z}\right) \in \mathcal{P}^{a, \bar{s}}$, and for a map $U: D(\sigma, r) \times \Sigma \rightarrow \mathcal{P}^{a, \bar{s}}$, define its Lipschitz seminorm $|U|_{r}^{\mathcal{L}}:=\sup _{\xi \neq \zeta} \frac{\left|\Delta_{\xi \zeta} U\right|_{r}}{|\xi-\zeta|}$, where $\Sigma$ is the parameter set $\Omega \times[0,1]^{n+1}, \Delta_{\xi \zeta} U=U(\cdot, \xi)-$ $U(\cdot, \zeta)$, and the supremum is taken over $\Sigma$. Denote by $X_{P}$ the vector field corresponding the Hamiltonian $P$ with respect to the symplectic structure $d x^{\prime} \wedge d y+\mathrm{i} d Z \wedge d \bar{Z}$, namely, $X_{P}=\left(\partial_{y} P,-\partial_{x^{\prime}} P,-\mathrm{i} \nabla_{\bar{Z}} P, \mathrm{i} \nabla_{Z} P\right)$. The proof of Lemma 3.7 can be found in 18 . Here, we omit it.

Lemma 3.7. The perturbation $P\left(x^{\prime}, y, Z, \bar{Z} ; \zeta\right)$ is real analytic for the real argument $\left(x^{\prime}, y, Z, \bar{Z}\right) \in D(2 \sigma, 2 r)$ and some given $\sigma, r>0$. And $P$ is Lipschitz for the parameter $\zeta \in \Sigma$. For each $\zeta \in \Sigma$, the gradients of $P$ with respect to $Z, \bar{Z}$ satisfy $\partial_{Z} P, \partial_{\bar{Z}} P \in$ $\mathcal{A}\left(l_{n+1}^{a, s}, l_{n+1}^{a, s+1 / 2}\right)$. In addition, for the perturbed term $P$, we have the following estimates $\sup _{D(\sigma, r) \times \Sigma}\left|X_{P}\right|_{r} \leq C \varepsilon^{8 / 3}$ and $\sup _{D(\sigma, r) \times \Sigma}\left|\partial_{\zeta} X_{P}\right|_{r} \leq C \varepsilon^{8 / 3}$, where $\sigma=\sigma_{1} / 4$ and $r=\varepsilon / 2$. 


\section{A KAM theorem with application to Hamiltonian (3.37)}

In order to prove our main result (Theorem 2.1), we apply an existing KAM theorem 8, 10 which is stated below.

Consider the perturbations of a family of linear integrable Hamiltonian

$$
H_{0}=\sum_{j=1}^{n^{\prime}} \widehat{\omega}_{j}(\xi) y_{j}+\frac{1}{2} \sum_{j=n^{\prime}+1}^{\infty} \widehat{\Omega}_{j}(\xi)\left(u_{j}^{2}+v_{j}^{2}\right),
$$

in $n^{\prime}$-dimensional angle-action coordinates $\left(x^{\prime}, y\right)$ and infinite-dimensional Cartesian coordinates $(u, v)$ with symplectic structure $\sum_{j=1}^{n^{\prime}} d x_{j}^{\prime} \wedge d y_{j}+\sum_{j=n^{\prime}+1}^{\infty} d u_{j} \wedge d v_{j}$, where $n^{\prime}>0$ is an integer. The tangent frequencies $\widehat{\omega}=\left(\widehat{\omega}_{1}, \ldots, \widehat{\omega}_{n^{\prime}}\right)$ and normal ones $\widehat{\Omega}=$ $\left(\widehat{\Omega}_{n^{\prime}+1}, \widehat{\Omega}_{n^{\prime}+2}, \ldots\right)$ depend on $n^{\prime}$ parameters $\xi \in \Pi \subset \mathbb{R}^{n^{\prime}}$, with $\Pi$ a closed bounded set of positive Lebesgue measure.

For each $\xi$ there is an invariant $n^{\prime}$-torus $\mathcal{T}_{0}^{n^{\prime}}=\mathbb{T}^{n^{\prime}} \times\{0,0,0\}$ with frequencies $\widehat{\omega}(\xi)$. In its normal space described by the $u v$-coordinates the origin is an elliptic fixed point with characteristic frequencies $\widehat{\Omega}(\xi)$. Hence $\mathcal{T}_{0}^{n^{\prime}}$ is linear stable. The aim is to prove the persistence of a large portion of this family of linearly stable rotational tori under small perturbations $H=H_{0}+P$ of $H_{0}$. To this end the following assumptions are made.

Assumption A. (Non-degeneracy). The map $\xi \mapsto \widehat{\omega}(\xi)$ is a Lipeomorphism between $\Pi$ and its image, that is, a homomorphism which is Lipschitz continuous in both directions. Moreover, for all integer vectors $(k, l) \in \mathbb{Z}^{n^{\prime}} \times \mathbb{Z}^{\infty}$ with $1 \leq|l| \leq 2$, meas $\{\xi:\langle k, \widehat{\omega}(\xi)\rangle+$ $\langle l, \widehat{\Omega}(\xi)\rangle=0\}=0$ and $\langle l, \widehat{\Omega}(\xi)\rangle \neq 0$ on $\Pi$.

Assumption B. (Spectral asymptotics and the Lipschitz property). There exist $\varsigma \geq 1$ and $\delta<\varsigma-1$ such that $\widehat{\Omega}_{j}(\xi)=j^{\varsigma}+\cdots+O\left(j^{\delta}\right)$, where the dots stand for fixed lower order term in $j$, allowing also negative exponents. More precisely, there exists a fixed, parameter-independent sequence $\bar{\Omega}$ with $\bar{\Omega}_{j}=j^{\varsigma}+\cdots$ such that the tails $\widehat{\Omega}_{j}-\bar{\Omega}_{j}$ give rise to a Lipschitz map $\widehat{\Omega}_{j}-\bar{\Omega}_{j}: \Pi \rightarrow l_{\infty}^{-\delta}$, where $l_{\infty}^{p}$ is the space of all real sequences with the finite norm $|w|_{p}=\sup _{j}\left|w_{j}\right| j^{p}$.

Assumption C. (Regularity). The perturbation $P(x, y, u, v ; \xi)$ is real analytic for real argument $(x, y, u, v) \in D(\sigma, r)$ for given $\sigma, r>0$, and Lipschitz in the parameters $\xi \in \Pi$, and for each $\xi \in \Pi$ its gradients with respect to $u, v$ satisfy

$$
P_{u}, P_{v} \in \mathcal{A}\left(l^{a, p}, l^{a, \bar{p}}\right), \quad \begin{cases}\bar{p} \geq p & \text { for } \varsigma>1 \\ \bar{p}>p & \text { for } \varsigma=1\end{cases}
$$

We assume that

$$
\left.|\widehat{\omega}|\right|_{\Pi} ^{\mathcal{L}}+|\widehat{\Omega}|_{-\delta, \Pi}^{\mathcal{L}} \leq M<\infty, \quad\left|(\widehat{\omega})^{-1}\right| \widehat{\mathcal{L}}(\Pi) \leq L<\infty
$$


In addition, we introduce the notations $\langle l\rangle_{\varsigma}=\max \left(1,\left|\sum_{j} j^{\varsigma} l_{j}\right|\right), A_{k}=1+|k|^{\tau}$, where $\tau>n^{\prime}+1$ is fixed. Finally, let $\mathcal{Z}=\{(k, l) \neq 0,|l| \leq 2\} \subset \mathbb{Z}^{n^{\prime}} \times \mathbb{Z}^{\infty}$.

Theorem 4.1. [10, Theorem A] Suppose that $H=H_{0}+P$ satisfies Assumptions A, B, and $\mathrm{C}$, and

$$
\epsilon=\sup _{D(\sigma, r) \times \Pi}\left|X_{P}\right|_{r}+\sup _{D(\sigma, r) \times \Pi} \frac{\alpha}{M}\left|X_{P}\right|_{r}^{\mathcal{L}} \leq \gamma \alpha
$$

where $0<\alpha \leq 1$ is a parameter, and $\gamma$ depends on $n^{\prime}, \tau$, and $\sigma$. Then there is a Cantor set $\Pi_{\alpha} \subset \Pi$ with meas $\left(\Pi \backslash \Pi_{\alpha}\right) \rightarrow 0$ as $\alpha \rightarrow 0$, a Lipschitz continuous family of torus embedding $\Phi: \mathbb{T}^{n^{\prime}} \times \Pi_{\alpha} \rightarrow \mathcal{P}^{a, \bar{p}}$, and a Lipschitz continuous map $\widetilde{\omega}: \Pi_{\alpha} \rightarrow \mathbb{R}^{n^{\prime}}$, such that for each $\xi \in \Pi_{\alpha}$ the map $\Phi$ restricted to $\mathbb{T}^{n^{\prime}} \times\{\xi\}$ is a real analytic embedding of an elliptic rotational torus with frequencies $\widetilde{\omega}(\xi)$ for the Hamiltonian $H$ at $\xi$.

Each embedding is real analytic on $|\operatorname{Im} x|<\sigma / 2$, and

$$
\left|\Phi-\Phi_{0}\right|_{r}+\frac{\alpha}{M}\left|\Phi-\Phi_{0}\right|_{r}^{\mathcal{L}} \leq \frac{c \epsilon}{\alpha}, \quad|\widetilde{\omega}-\widehat{\omega}|+\frac{\alpha}{M}|\widetilde{\omega}-\widehat{\omega}|^{\mathcal{L}} \leq c \epsilon,
$$

uniformly on that domain and $\Pi_{\alpha}$, where $\Phi_{0}: \mathbb{T}^{n^{\prime}} \times \Pi \rightarrow \mathcal{T}_{0}^{n^{\prime}}$ is the trivial embedding, and $c \leq \gamma^{-1}$ depends on the same parameters as $\gamma$.

Moreover, there exist Lipschitz maps $\mu_{j}$ and $\Lambda_{j}$ on $\Pi$ for $j \geq 0$ satisfying $\mu_{0}=\widehat{\omega}$, $\Lambda_{0}=\widehat{\Omega}$ and

$$
\left|\mu_{j}-\widehat{\omega}\right|+\frac{\alpha}{M}\left|\mu_{j}-\widehat{\omega}\right|^{\mathcal{L}} \leq c \epsilon, \quad\left|\Lambda_{j}-\widehat{\Omega}\right|_{-\delta}+\frac{\alpha}{M}\left|\Lambda_{j}-\widehat{\Omega}\right|_{-\delta}^{\mathcal{L}} \leq c \epsilon,
$$

such that $\Pi \backslash \Pi_{\alpha} \subset \bigcup \mathcal{R}_{k, l}^{j}(\alpha)$, where

$$
\mathcal{R}_{k, l}^{j}(\alpha)=\left\{\xi \in \Pi:\left|\left\langle k, \mu_{j}(\xi)\right\rangle+\left\langle l, \Lambda_{j}(\xi)\right\rangle\right|<\alpha \frac{\langle l\rangle_{d}}{A_{k}}\right\},
$$

and the union is taken over all $j \geq 0$ and $(k, l) \in \mathcal{Z}$ such that $|k|>K_{1} 2^{j-1}$ for $j \geq 1$ with a constant $K_{1} \geq 1$ depending only on $n^{\prime}$ and $\tau$.

Theorem 4.2. [10, Theorem D] Suppose that in Theorem 4.1 the unperturbed frequencies are affine functions of the parameters. Then there is a constant $\widetilde{c}$ such that

$$
\operatorname{meas}\left(\Pi \backslash \Pi_{\alpha}\right) \leq \widetilde{c}(\operatorname{diam} \Pi)^{n^{\prime}-1} \alpha^{\widetilde{\mu}}, \quad \widetilde{\mu}= \begin{cases}1 & \text { for } \varsigma>1, \\ \frac{\kappa}{\kappa+1-(\varpi / 4)} & \text { for } \varsigma=1,\end{cases}
$$

for all sufficiently small $\alpha$, where $\varpi$ is any number in $[0, \min (\bar{p}-p, 1))$, and in the case $\varsigma=1, \kappa$ is a positive constant such that $\frac{\widehat{\Omega}_{i}-\widehat{\Omega}_{j}}{i-j}=1+\mathcal{O}\left(j^{-\kappa}\right), i>j$ uniformly on $\Pi$.

Remark 4.3. By Remarks in 16, $\widetilde{c}=c_{1} L^{n^{\prime}} M^{n^{\prime}-1}$ where $c_{1}$ is an absolute constant. 
In order to apply the above theorems to our problem, we need to introduce a new parameter $\bar{\omega}$. For any $\omega_{-} \in \Omega$ fixed and $\omega \in \overline{\bar{\Omega}}:=\left\{\omega \in \Omega|| \omega-\omega_{-} \mid \leq \varepsilon\right\}$, suppose $\omega=\omega_{-}+\varepsilon \bar{\omega}, \bar{\omega} \in[0,1]^{m}$. The Hamiltonian (3.37) becomes

$$
H=\langle\widehat{\omega}(\xi), y\rangle+\langle\widehat{\Omega}(\xi), \widehat{Z}\rangle+P,
$$

where

$$
\widehat{\omega}(\xi)=\omega \oplus \breve{\omega}, \quad \widehat{\Omega}(\xi)=\widetilde{\beta}+\varepsilon^{2} B \widetilde{\xi}
$$

$B=\left(\bar{G}_{i j}\right)_{0 \leq j \leq n<i}, \breve{\omega}=\widetilde{\alpha}+\varepsilon^{2} A \widetilde{\xi}, A=\left(\bar{G}_{i j}\right)_{0 \leq i, j \leq n}, \xi=\bar{\omega} \oplus \widetilde{\xi}, \widehat{Z}=\left(\left|z_{n+1}\right|^{2},\left|z_{n+2}\right|^{2}, \ldots\right)$, $y=J \oplus \widetilde{\rho}, \widetilde{\alpha}=\left(\sqrt{\lambda_{0}}, \sqrt{\lambda_{1}}, \ldots, \sqrt{\lambda_{n}}\right)$, and $\widetilde{\beta}=\left(\sqrt{\lambda_{n+1}}, \sqrt{\lambda_{n+2}}, \ldots\right)$. So $\breve{\omega}_{j}(0 \leq j \leq n)$ can be written as

$$
\breve{\omega}_{j}=\sqrt{\lambda_{j}}+\varepsilon^{2} \sum_{i=0}^{n} A_{j i} \widetilde{\xi}_{i} \quad \text { and } \quad A_{i j}= \begin{cases}\frac{3 g_{0}}{4 \pi \sqrt{\lambda_{i} \lambda_{j}}} & \text { if } i \neq j, \\ \frac{9 g_{0}}{16 \pi \lambda_{i}} & \text { if } i=j \neq 0 \\ \frac{3 g_{0}}{8 \pi \lambda_{0}} & \text { if } i=j=0 .\end{cases}
$$

Furthermore, we calculate and get that

$$
\operatorname{det} A=\frac{3 g_{0}}{4 \pi \lambda_{0} \lambda_{1} \cdots \lambda_{n}} \cdot\left(1+\sum_{i=0}^{n} \frac{1}{a_{i}-1}\right)\left(a_{0}-1\right)\left(a_{1}-1\right) \cdots\left(a_{n}-1\right) \neq 0
$$

where $a_{0}=1 / 2$ and $a_{i}=3 / 4(1 \leq i \leq n)$. The proof of the following lemma is as same as Lemma 3.7 .

Lemma 4.4. Setting $\Pi=[0,1]^{m+n+1}$, we have $X_{P} \in \mathcal{A}\left(l_{n+1}^{a, s}, l_{n+1}^{a, s+1 / 2}\right)$, $\sup _{D(\sigma, r) \times \Pi}\left|X_{P}\right|_{r} \leq$ $C \varepsilon^{8 / 3}$ and $\sup _{D(\sigma, r) \times \Pi}\left|\partial_{\zeta} X_{P}\right|_{r} \leq C \varepsilon^{8 / 3}$.

We introduce $z_{j}=\frac{1}{\sqrt{2}}\left(u_{j}+\mathrm{i} v_{j}\right)$. The normal form 4.3 becomes

$$
H=\langle\widehat{\omega}(\xi), y\rangle+\frac{1}{2}\left\langle\widehat{\Omega}(\xi), u^{2}+v^{2}\right\rangle+P
$$

with respect to the symplectic structure $\sum_{0 \leq j \leq n+m} d x_{j} \wedge d y_{j}+\sum_{j \geq n+m+1} d u_{j} \wedge d v_{j}$. We only need to verify Assumptions $\mathrm{A}, \mathrm{B}$, and $\mathrm{C}$ for 4.5 .

Note that $\operatorname{det} A \neq 0$ and $\min _{0 \leq i<n}\left|j_{i+1}-j_{i}\right| \leq n$. According to the same method in [18, p. 2205], the Assumption A is verified. Assumption B is fulfilled for $\widehat{\Omega}$ with $\delta=-1$ and $\varsigma=1$. Assumption $\mathrm{C}$ holds by using of Lemma 4.4 letting $\bar{p}=s+1 / 2$ and $p=s$. Furthermore, from 4.4, 4.1) is satisfied letting $M=C \varepsilon$ and $L=C \varepsilon^{-1}$. Setting $\alpha=\varepsilon^{2}$ and by Lemma 4.4, the smallness condition (4.2) is verified. Due to $\kappa=2$, we can take $\widetilde{\mu}=24 / 35$.

Applying Theorems 4.1 and 4.2 for Hamiltonian (4.5), the proof of Theorem 2.1 is completed. 


\section{Appendix}

Proof of Lemma 3.2. When $i, j, d, l$ are all non-zero integers, from [11], (3.7) holds true. Thus we only discuss the case that at least one of $i, j, d, l$ is zero. Without loss of generality, we assume that $|i| \leq|j| \leq|d| \leq|l|$. Then, (3.6) reduces to $|i|-|j|-|d|+|l|=0$ and $|i|+|j|+|d|-|l|=0$. Now we distinguish $\delta=\lambda_{i}^{\prime}+\lambda_{j}^{\prime}+\lambda_{d}^{\prime}+\lambda_{l}^{\prime}$ according to the number of minus signs. For convenience, we let for example $\delta_{+++-}=+\sqrt{\lambda_{|i|}}+\sqrt{\lambda_{|j|}}+\sqrt{\lambda_{|d|}}-\sqrt{\lambda_{|l|}}$. It is similar for all the other combinations of plus and minus signs.

Case 1. All of $i, j, d, l$ are 0 or -0 . Since $(i, j, d, l) \notin \mathcal{N}_{n},(i, j, d, l) \not \equiv(0,-0,0,-0)$. So $\left|\lambda_{i}^{\prime}+\lambda_{j}^{\prime}+\lambda_{d}^{\prime}+\lambda_{l}^{\prime}\right|=4 \sqrt{\mu}$ or $2 \sqrt{\mu}$.

Case 2. Three of $i, j, d, l$ are 0 or -0 . This case can reduce to Case 1 because of (3.6).

Case 3. Two of $i, j, d, l$ are 0 or -0 . Without loss of generality, we assume $i$ and $j$ are 0 or -0 .

Case 3.1. When $(i, j) \equiv(0,0),|d|=|l|$ because of $(3.6)$. When $d=-l, \mid \lambda_{i}^{\prime}+\lambda_{j}^{\prime}+$ $\lambda_{d}^{\prime}+\lambda_{l}^{\prime} \mid=2 \sqrt{\mu}$. When $d=l>0,\left|\lambda_{i}^{\prime}+\lambda_{j}^{\prime}+\lambda_{d}^{\prime}+\lambda_{l}^{\prime}\right| \geq 2 \sqrt{\mu}+2 \sqrt{1+\mu}$. When $d=l<0$, $\left|\lambda_{i}^{\prime}+\lambda_{j}^{\prime}+\lambda_{d}^{\prime}+\lambda_{l}^{\prime}\right| \geq 2 \sqrt{1+\mu}-2 \sqrt{\mu}$.

Case 3.2. When $(i, j) \equiv(-0,-0),|d|=|l|$ because of $(3.6)$. When $d=-l, \mid \lambda_{i}^{\prime}+\lambda_{j}^{\prime}+$ $\lambda_{d}^{\prime}+\lambda_{l}^{\prime} \mid=2 \sqrt{\mu}$. When $d=l>0,\left|\lambda_{i}^{\prime}+\lambda_{j}^{\prime}+\lambda_{d}^{\prime}+\lambda_{l}^{\prime}\right| \geq 2 \sqrt{1+\mu}-2 \sqrt{\mu}$. When $d=l<0$, $\left|\lambda_{i}^{\prime}+\lambda_{j}^{\prime}+\lambda_{d}^{\prime}+\lambda_{l}^{\prime}\right| \geq 2 \sqrt{\mu}+2 \sqrt{1+\mu}$.

Case 3.3. When $(i, j) \equiv(-0,0),|d|=|l|$ because of $(3.6)$. Since $(i, j, d, l) \notin \mathcal{N}_{n}$, $d \neq-l$. So we only consider the case $d=l$. When $d=l,\left|\lambda_{i}^{\prime}+\lambda_{j}^{\prime}+\lambda_{d}^{\prime}+\lambda_{l}^{\prime}\right|=2 \sqrt{d^{2}+\mu} \geq$ $2 \sqrt{1+\mu}$.

Case 4 . When one of $i, j, d, l$ is 0 or -0 , without loss of generality, we assume

$$
i=0 \text { or } \quad-0, \quad \text { and } \quad 0=|i|<1 \leq|j| \leq|d| \leq|l| .
$$

By using of (5.1), (3.6) reduces to

$$
|l|=|j|+|d| .
$$

Now we discuss $\delta$ according to the number of minus signs.

Case 4.0. No minus sign. $\delta_{++++} \geq \sqrt{\mu}+3 \sqrt{\mu+1}$.

Case 4.1. One minus sign. It is clear that $\delta_{++-+}, \delta_{+-++} \geq \sqrt{1+\mu}+\sqrt{\mu}$ and $\delta_{-+++}>$ $2 \sqrt{1+\mu}$ hold. The remaining is $\delta_{+++-}$. We consider the function $\delta_{2}(\mu)=\sqrt{\mu}+\sqrt{j^{2}+\mu}+$ $\sqrt{d^{2}+\mu}-\sqrt{l^{2}+\mu}$, where $j, d \geq 1$. From $(5.2), \delta_{2}(0)=0$ and

$$
\delta_{2}^{\prime}(\mu)=\frac{1}{2}\left(\frac{1}{\sqrt{\mu}}+\frac{1}{\sqrt{j^{2}+\mu}}+\frac{1}{\sqrt{d^{2}+\mu}}-\frac{1}{\sqrt{(j+d)^{2}+\mu}}\right) \geq \frac{1}{2 \sqrt{\mu}}>0 .
$$

Hence, $\delta_{2}(\mu)$ is strictly monotone increasing in $\mu$ and we have that

$$
\delta_{2}(\mu)=\int_{0}^{\mu} \delta_{2}^{\prime}(\mu) d \mu \geq \int_{0}^{\mu} \frac{1}{2 \sqrt{\mu}} d \mu=\sqrt{\mu} .
$$


Case 4.2. Two minus signs. Since $\delta_{-+-+}, \delta_{--++} \geq \delta_{+--+},\left|\delta_{+-+-}\right|=\left|\delta_{-+-+}\right|$, $\left|\delta_{++--}\right|=\left|\delta_{--++}\right|$and $\left|\delta_{-++-}\right|=\left|\delta_{+--+}\right|$, it suffices to discuss the case $\delta_{+--+}$. From $(5.2), \delta_{+--+}=\sqrt{\mu}-\sqrt{|j|^{2}+\mu}-\sqrt{|d|^{2}+\mu}+\sqrt{(|j|+|d|)^{2}+\mu}$, where $j, d \leq-1$.

We suppose that $f_{d}(x):=\sqrt{(x+|d|)^{2}+\mu}-\sqrt{x^{2}+\mu}$ for $x \geq 0$, where $d \leq-1$ is fixed. So

$$
f_{d}^{\prime}(x)=\frac{(|d|+x) \sqrt{x^{2}+\mu}-x \sqrt{(|d|+x)^{2}+\mu}}{\sqrt{(|d|+x)^{2}+\mu} \sqrt{x^{2}+\mu}} .
$$

Now we compare $(|d|+x) \sqrt{x^{2}+\mu}$ with $x \sqrt{(|d|+x)^{2}+\mu}$. Since

$$
\left[(|d|+x) \sqrt{x^{2}+\mu}\right]^{2}-\left[x \sqrt{(|d|+x)^{2}+\mu}\right]^{2} \geq \mu d^{2} \geq \mu>0,
$$

$(|d|+x) \sqrt{x^{2}+\mu}>x \sqrt{(|d|+x)^{2}+\mu}$ holds true. This yields that $f_{d}^{\prime}>0$ for $x \geq 0$. So, $f_{d}$ is strictly monotone increasing as $x \geq 0$ which implies that $f_{d}(|j|) \geq f_{d}(1)>f_{d}(0)$ if $j \leq-1$. It follows that $f_{d}(|j|)-f_{d}(0) \geq f_{d}(1)-f_{d}(0)>0$, which means

$$
\delta_{+--+}=f_{d}(|j|)-f_{d}(0) \geq \sqrt{(|d|+1)^{2}+\mu}-\sqrt{1+\mu}-\sqrt{|d|^{2}+\mu}+\sqrt{\mu} .
$$

In addition, we assume that $f_{1}(x)=\sqrt{(x+1)^{2}+\mu}-\sqrt{x^{2}+\mu}$. Similar to the above discussion, we can prove that $f_{1}$ is strictly monotone increasing as $x \geq 0$. Thus, we have that $f_{1}(|d|)>f_{1}(1 / 2)>f_{1}(0)$ as $d \leq-1$. So $f_{1}(|d|)-f_{1}(0)>f_{1}(1 / 2)-f_{1}(0)>0$ which means that

$$
\begin{aligned}
& \sqrt{(|d|+1)^{2}+\mu}-\sqrt{1+\mu}-\sqrt{|d|^{2}+\mu}+\sqrt{\mu} \\
> & \sqrt{(3 / 2)^{2}+\mu}-\sqrt{(1 / 2)^{2}+\mu}-\sqrt{1+\mu}+\sqrt{\mu}>0 .
\end{aligned}
$$

Therefore, it is derived from (5.3) and (5.4) that $\delta_{+--+}>\sqrt{(3 / 2)^{2}+\mu}-\sqrt{(1 / 2)^{2}+\mu}-$ $\sqrt{1+\mu}+\sqrt{\mu}>0$.

Case 4.3. Three minus signs. This case can be reduced to Case 4.1.

Case 4.4. Four minus signs. This case can be reduced to Case 4.0.

All of the above, these lower bounds give the claimed estimate. The proof is completed.

Proof of Lemma 3.4. Assume

$$
\mathcal{R}_{i j d l, k}^{2}=\left\{\omega \in[\varrho, 2 \varrho]^{m}:\left|\lambda_{i}^{\prime}+\lambda_{j}^{\prime}+\lambda_{d}^{\prime}+\lambda_{l}^{\prime}+\langle k, \omega\rangle\right|<\frac{\varrho \varepsilon^{1 / 3}}{|k|^{m+3}}\right\}
$$

and $\Omega^{2}=\bigcup_{|k| \geq 1} \bigcup_{i, j, d, l} \mathcal{R}_{i j d l, k}^{2}$. Consider two hyperplanes $\lambda_{i}^{\prime}+\lambda_{j}^{\prime}+\lambda_{d}^{\prime}+\lambda_{l}^{\prime}+\langle k, \omega\rangle=$ $\pm \frac{\varrho \varepsilon^{1 / 3}}{|k|^{m+3}}$. We have

$$
\text { meas } \mathcal{R}_{i j d l, k}^{2} \leq m|k|^{-1}(\sqrt{2} \varrho)^{m-1} \frac{2 \varrho \varepsilon^{1 / 3}}{|k|^{m+3}} \leq \frac{2(\sqrt{2})^{m-1} m \varepsilon^{1 / 3}}{|k|^{m+3}|k|} \varrho^{m} \leq C \frac{\varrho^{m} \varepsilon^{1 / 3}}{|k|^{m+3}|k|}
$$


where $C$ depends on $m$.

Assume $\lambda_{i}^{\prime}+\lambda_{j}^{\prime}+\lambda_{d}^{\prime}+\lambda_{l}^{\prime} \neq 0, k \neq 0$ and $(i, j, d, l) \in \mathcal{L}_{n}$. Now we let $|i|=$ $\min \{|i|,|j|,|d|,|l|\}$. The other cases can be discussed in the same way. It is evident that $0 \leq|i| \leq n$.

Case 1. If $j, d, l$ have same sign, we can assume $f_{j d l}=\operatorname{sgn} j \cdot\left(\sqrt{\lambda_{|j|}}+\sqrt{\lambda_{|d|}}+\sqrt{\lambda_{|l|}}\right)$. Let

$$
\mathcal{R}_{i j d l, k}^{2,1}=\left\{\omega \in[\varrho, 2 \varrho]^{m}:\left|f_{j d l}+\lambda_{i}^{\prime}+\langle k, \omega\rangle\right|<\frac{\varrho \varepsilon^{1 / 3}}{|k|^{m+3}}\right\}
$$

and $\Omega^{2,1}=\bigcup_{|k| \geq 1} \bigcup_{i, j, d, l} \mathcal{R}_{i j d l, k}^{2,1}$. Clearly,

$$
\left|\lambda_{i}^{\prime}+\langle k, \omega\rangle\right| \leq 2 \sqrt{n^{2}+\mu}+2|k||\omega|
$$

by using of $0 \leq|i| \leq n$. So, when $\left|f_{j d l}\right|>3 \sqrt{n^{2}+\mu}+2|k||\omega|$, we have

$$
\left|f_{j d l}+\lambda_{i}^{\prime}+\langle k, \omega\rangle\right|>\left|f_{j d l}\right|-\left|\lambda_{i}^{\prime}+\langle k, \omega\rangle\right|>\sqrt{n^{2}+\mu} \geq \frac{\varrho \varepsilon^{1 / 3}}{|k|^{m+3}},
$$

when $\varepsilon$ is small enough. This implies that the set $\mathcal{R}_{i j d l, k}^{2,1}$ is empty. Therefore, we only need to consider the case that $j, d, l \leq 3 \sqrt{n^{2}+\mu}+2|k||\omega|$ in order to calculate $\Omega^{2,1}$. It yields that, from (5.5) and 3.35,

$$
\begin{aligned}
\operatorname{meas} \Omega^{2,1} & =\operatorname{meas} \bigcup_{|k| \geq 1} \bigcup_{i, j, d, l} \mathcal{R}_{i j d l, k}^{2,1} \\
& =\operatorname{meas} \bigcup_{|k| \geq 1} \bigcup_{0 \leq|i| \leq n} \mathcal{R}_{i j|,| d|,| l \mid \leq 3}^{2} \sqrt{n^{2}+\mu}+2|k||\omega| \\
& \leq \sum_{|k| \geq 1} 2(n+1)\left(6 \sqrt{n^{2}+\mu}+4 \varrho|k|+2\right)^{3} \frac{C \varrho^{m} \varepsilon^{1 / 3}}{|k|^{m+3}|k|} \\
& \leq C \varrho^{m} \varepsilon^{1 / 3} \sum_{|k| \geq 1} \frac{1}{|k|^{m+1}} \leq C \varrho^{m} \varepsilon^{1 / 3} \sum_{|k|=l \geq 1} \frac{1}{l^{m+1}} 2^{m} l^{m-1} \\
& =C 2^{m} \varrho^{m} \varepsilon^{1 / 3} \sum_{l \geq 1} \frac{1}{l^{2}} \leq C \varrho^{m} \varepsilon^{1 / 3},
\end{aligned}
$$

where the constant $C$ depends on $\mu, n, \varrho$ and $m$.

Case 2. Assume $j, d$ and $l$ have different signs. Without loss of generality, we let that $j, d$ have the same sign, and $l$ have the different sign. So, $f_{j d l}=\operatorname{sgn} j \cdot\left(\sqrt{\lambda_{|j|}}+\sqrt{\lambda_{|d|}}-\right.$ $\left.\sqrt{\lambda_{|l|}}\right):=\widetilde{f}_{j d}+\operatorname{sgn} l \cdot \sqrt{\lambda_{|l|}}$. The other cases can be discussed in the same way. Now, it suffices to consider the two cases as follows.

Case 2.1. Suppose that $|i| \leq|l| \leq \tilde{N}$,

$$
\mathcal{R}_{i j d l, k}^{2,2}=\left\{\omega \in[\varrho, 2 \varrho]^{m}:\left|\widetilde{f}_{j d}+\operatorname{sgn} l \cdot \sqrt{\lambda_{|l|}}+\lambda_{i}^{\prime}+\langle k, \omega\rangle\right|<\frac{\varrho \varepsilon^{1 / 3}}{|k|^{m+5}},|l| \leq \widetilde{N}\right\}
$$


and $\Omega^{2,2}=\bigcup_{|k| \geq 1} \bigcup_{i, j, d, l} \mathcal{R}_{i j d l, k}^{2,2}$, where

$$
\widetilde{N}=\widetilde{N}_{1}|k|, \quad \widetilde{N}_{1}=\sqrt{n^{2}+\mu}+2 \varrho+2 \sqrt{\mu}+2 .
$$

Then

$$
\left|\operatorname{sgn} l \cdot \sqrt{\lambda_{|l|}}+\lambda_{i}^{\prime}+\langle k, \omega\rangle\right| \leq 3 \sqrt{\tilde{N}^{2}+\mu}+|k||\omega| .
$$

When $\left|\tilde{f}_{j d}\right|>4 \sqrt{\tilde{N}^{2}+\mu}+|k||\omega|$, it follows that, as $\varepsilon$ small enough,

$$
\begin{aligned}
\left|\tilde{f}_{j d}+\operatorname{sgn} l \cdot \sqrt{\lambda_{|l|}}+\lambda_{i}^{\prime}+\langle k, \omega\rangle\right| & >\left|\tilde{f}_{j d}\right|-\left|\operatorname{sgn} l \cdot \sqrt{\lambda_{|l|}}+\lambda_{i}^{\prime}+\langle k, \omega\rangle\right| \\
& \geq \sqrt{\tilde{N}^{2}+\mu}>\widetilde{N}>2>\frac{\varrho \varepsilon^{1 / 3}}{|k|^{m+3}},
\end{aligned}
$$

which implies that the set $\mathcal{R}_{i j d l, k}^{2,2}$ is empty. Therefore, we only need to consider the case that $j, d \leq 4 \sqrt{\widetilde{N}^{2}+\mu}+|k||\omega|$ in order to calculate $\Omega^{2,2}$. It yields that

$$
\begin{aligned}
& \text { meas } \Omega^{2,2}=\operatorname{meas} \bigcup_{|k| \geq 1} \bigcup_{i, j, d, l} \mathcal{R}_{i j d l, k}^{2,2} \\
& =\operatorname{meas} \bigcup_{|k| \geq 1} \bigcup_{0 \leq|i| \leq n} \bigcup_{|l| \leq \widetilde{N}} \bigcup_{|j|,|d| \leq 4} \mathcal{R}_{i j d l, k}^{2,2} \\
& \leq \sum_{|k| \geq 1} 4(n+1)(\tilde{N}+1)\left(8 \sqrt{\tilde{N}^{2}+\mu}+4 \varrho|k|+2\right)^{2} \frac{\varrho^{m} \varepsilon^{1 / 3}}{|k|^{m+3}|k|} \\
& \leq C \varrho^{m} \varepsilon^{1 / 3} \sum_{|k| \geq 1} \frac{1}{|k|^{m+1}} \leq C \varrho^{m} \varepsilon^{1 / 3} \sum_{|k|=l \geq 1} \frac{1}{l^{m+1}} 2^{m} l^{m-1} \\
& =C 2^{m} \varrho^{m} \varepsilon^{1 / 3} \sum_{l \geq 1} \frac{1}{l^{2}} \leq C \varrho^{m} \varepsilon^{1 / 3},
\end{aligned}
$$

where the constant $C$ depends on $\mu, n, \varrho$ and $m$.

Case 2.2. Suppose that $|l|>\widetilde{N}$,

$$
\mathcal{R}_{i j d l, k}^{2,3}=\left\{\omega \in[\varrho, 2 \varrho]^{m}:\left|\tilde{f}_{j d}+\operatorname{sgn} l \cdot \sqrt{\lambda_{|l|}}+\lambda_{i}^{\prime}+\langle k, \omega\rangle\right|<\frac{\varrho \varepsilon^{1 / 3}}{|k|^{m+3}},|l|>\widetilde{N}\right\}
$$

and $\Omega^{2,3}=\bigcup_{|k| \geq 1} \bigcup_{i, j, d, l} \mathcal{R}_{i j d l, k}^{2,3}$. We divide this case into two cases (a) and (b) below.

Case (a). If $|d|=|l|$, then $\sqrt{\lambda_{|d|}}=\sqrt{\lambda_{|l|}}$. We have

$$
\left|\tilde{f}_{j d}+\operatorname{sgn} l \cdot \sqrt{\lambda_{|l|}}+\lambda_{i}^{\prime}+\langle k, \omega\rangle\right|=\left|\operatorname{sgn} j \cdot \sqrt{\lambda_{|j|}}+\lambda_{i}^{\prime}+\langle k, \omega\rangle\right| \cdot
$$

If $|j| \geq \sqrt{n^{2}+\mu}+|k||\omega|+1$, then it follows that

$$
\begin{aligned}
\left|\tilde{f}_{j d}+\operatorname{sgn} l \cdot \sqrt{\lambda_{|l|}}+\lambda_{i}^{\prime}+\langle k, \omega\rangle\right| & \geq\left|\sqrt{\lambda_{|j|}}\right|-\left|\lambda_{i}^{\prime}+\langle k, \omega\rangle\right| \\
& >\sqrt{n^{2}+\mu}+|k||\omega|+1-\sqrt{n^{2}+\mu}-|k||\omega| \\
& \geq 1 \geq \frac{\varrho \varepsilon^{1 / 3}}{|k|^{m+5}},
\end{aligned}
$$


when $\varepsilon$ small enough. So, it is sufficient to consider the case $|j|<\sqrt{n^{2}+\mu}+|k||\omega|+1$. In fact,

$$
\begin{aligned}
\operatorname{meas} \Omega^{2,3} & =\operatorname{meas} \bigcup_{|k| \geq 1} \bigcup_{|j|<\sqrt{n^{2}+\mu}+|k||\omega|+1} \bigcup_{0 \leq|i| \leq n} \mathcal{R}_{i j d l, k}^{2,3} \\
& \leq \sum_{|k| \geq 1} 2(n+1)\left(\sqrt{n^{2}+\mu}+|k||\omega|+2\right) \frac{C \varrho^{m} \varepsilon^{1 / 3}}{|k||k|^{m+3}} \\
& \leq C \varrho^{m} \varepsilon^{1 / 3} \sum_{|k| \geq 1} \frac{1}{|k|^{m+3}} \leq C \varrho^{m} \varepsilon^{1 / 3} \sum_{|k|=l \geq 1} \frac{1}{l^{m+3}} 2^{m} l^{m-1} \\
& =C 2^{m} \varrho^{m} \varepsilon^{1 / 3} \sum_{l \geq 1} \frac{1}{l^{4}} \leq C \varrho^{m} \varepsilon^{1 / 3},
\end{aligned}
$$

where the constant $C$ depends on $n, \mu, \varrho$ and $m$. In the same way, we can get the same result in the case $|j|=|l|$.

Case (b). Suppose that $|d| \neq|l|$ and $|j| \neq|l|$. It is evident that

$$
\begin{aligned}
\left|\sqrt{\lambda_{|d|}}-\sqrt{\lambda_{|l|}}\right| & =|| d\left|+\frac{\mu}{\sqrt{d^{2}+\mu}+|d|}-\right| l\left|-\frac{\mu}{\sqrt{l^{2}+\mu}+|l|}\right| \\
& \geq|||d|-|l||-| \frac{\mu}{\sqrt{d^{2}+\mu}+|d|}+\frac{\mu}{\sqrt{l^{2}+\mu}+|l|}|| \\
& \geq|||d|-|l||-2 \sqrt{\mu}| .
\end{aligned}
$$

If $p=|| d|-| l||>\widetilde{N}$, then

$$
\begin{aligned}
& \left|\tilde{f}_{j d}+\operatorname{sgn} l \cdot \sqrt{\lambda_{|l|}}+\lambda_{i}^{\prime}+\langle k, \omega\rangle\right| \\
\geq & \sqrt{\lambda_{|d|}}-\sqrt{\lambda_{|l|}}-\sqrt{i^{2}+\mu}-2 \varrho|k|>\widetilde{N}-2 \sqrt{\mu}-\sqrt{n^{2}+\mu}-2 \varrho|k| \\
= & \left(\sqrt{n^{2}+\mu}+2 \varrho+2 \mu+2\right)|k|-2 \sqrt{\mu}-\sqrt{n^{2}+\mu}-2 \varrho|k| \geq 2 \geq \frac{\varrho \varepsilon^{1 / 3}}{|k|^{m+3}},
\end{aligned}
$$

which implies that the set $\mathcal{R}_{i j d l, k}^{2,3}$ is empty, when $\varepsilon$ is small enough. In the same way, we can get the same result if $q=|| j|-| l||>\tilde{N}$. So we only need to consider the case that $1 \leq p \leq \widetilde{N}$ and $1 \leq q \leq \widetilde{N}$

Case (b.1). Let $1 \leq p \leq \tilde{N}, 1 \leq q \leq \widetilde{N}$ and $|d|>\left(2 \widetilde{N}_{1}+1\right)|k|$. It follows that

$$
\begin{aligned}
& \left|\tilde{f}_{j d}+\operatorname{sgn} l \cdot \sqrt{\lambda_{|l|}}+\lambda_{i}^{\prime}+\langle k, \omega\rangle\right| \\
\geq & \sqrt{\lambda_{|d|}}-\left|\sqrt{\lambda_{|j|}}-\sqrt{\lambda_{|l|}}\right|-\left|\lambda_{i}^{\prime}+\langle k, \omega\rangle\right| \\
> & \left(2 \widetilde{N}_{1}+1\right)|k|-\left(\widetilde{N}_{1}|k|+2 \sqrt{\mu}\right)-\sqrt{n^{2}+\mu}-2 \varrho|k| \\
= & \left(\sqrt{n^{2}+\mu}+2 \varrho+2 \sqrt{\mu}+3\right)|k|-2 \sqrt{\mu}-\sqrt{n^{2}+\mu}-2 \varrho|k| \\
\geq & 3 \geq \frac{\varrho \varepsilon^{1 / 3}}{|k|^{m+3}},
\end{aligned}
$$


which implies that the set $\mathcal{R}_{i j d l, k}^{2,3}$ is empty, when $\varepsilon$ is small enough. In the same way, we can get the same result if $1 \leq p \leq \widetilde{N}, 1 \leq q \leq \widetilde{N}$ and $|j|>\left(2 \widetilde{N}_{1}+1\right)|k|$ hold. So we only leave the following case.

Case (b.2). Let $1 \leq p \leq \widetilde{N}, 1 \leq q \leq \widetilde{N}, 0 \leq|d| \leq\left(2 \widetilde{N}_{1}+1\right)|k|$ and $0 \leq|j| \leq$ $\left(2 \widetilde{N}_{1}+1\right)|k|$. In this case, it can be reduced that $0 \leq|l| \leq\left(3 \widetilde{N}_{1}+1\right)|k|$. So,

$$
\begin{aligned}
\operatorname{meas} \Omega^{2,3} & =\operatorname{meas} \bigcup_{|k| \geq 1} \bigcup_{0 \leq|l| \leq\left(3 \widetilde{N}_{1}+1\right)|k|} \bigcup_{0 \leq|j|,|d| \leq\left(2 \widetilde{N}_{1}+1\right)} \bigcup_{|k|} \mathcal{R}_{i j d l, k}^{2,3} \\
& \leq \sum_{|k| \geq 1} 2(n+1)\left(\left(4 \widetilde{N}_{1}+2\right)|k|+1\right)^{2}\left(\left(6 \widetilde{N}_{1}+2\right)|k|+1\right) \frac{C \varrho^{m} \varepsilon^{1 / 3}}{|k||k|^{m+3}} \\
& \leq C \varrho^{m} \varepsilon^{1 / 3} \sum_{|k| \geq 1} \frac{1}{|k|^{m+1}} \leq C \varrho^{m} \varepsilon^{1 / 3} \sum_{|k|=l \geq 1} \frac{1}{l^{m+1}} 2^{m} l^{m-1} \\
& =C 2^{m} \varrho^{m} \varepsilon^{1 / 3} \sum_{l \geq 1} \frac{1}{l^{2}} \leq C \varrho^{m} \varepsilon^{1 / 3},
\end{aligned}
$$

where the constant $C$ depends on $n, \mu, \varrho$ and $m$.

In the same way, we can get the similar results in the cases $|j|,|d|$ or $|l|=\min \{|i|,|j|$, $|d|,|l|\}$ wherein we can construct sets similar to $\Omega^{2,1}, \Omega^{2,2}$ and $\Omega^{2,3}$, etc. Assume $\Omega^{2}$ is the union of all the sets (the number of the sets is obviously finite). Thus, there is a constant $C_{2}$ such that meas $\Omega^{2} \leq C_{2} \varrho^{m} \varepsilon^{1 / 3}$, where $C_{2}$ depends on $\mu, n, \varrho$ and $m$. Finally, we suppose $\bar{\Omega}=[\varrho, 2 \varrho]^{m} \backslash \Omega^{2}$, which satisfies that meas $\bar{\Omega} \geq\left(1-C_{2} \varepsilon^{1 / 3}\right) \varrho^{m}$. The proof is completed.

\section{Acknowledgments}

The author would like to thank the referees for their valuable comments and suggestions which have helped to improve this paper and inspired our future work.

\section{References}

[1] D. Bambusi, M. Berti and E. Magistrelli, Degenerate KAM theory for partial differential equations, J. Differential Equations 250 (2011), no. 8, 3379-3397.

[2] M. Berti, L. Biasco and M. Procesi, Existence and stability of quasi-periodic solutions for derivative wave equations, Atti Accad. Naz. Lincei Rend. Lincei Mat. Appl. 24 (2013), no. 2, 199-214.

[3] M. Berti and P. Bolle, Sobolev quasi-periodic solutions of multidimensional wave equations with a multiplicative potential, Nonlinearity 25 (2012), no. 9, 2579-2613. 
[4] L. Chierchia and J. You, KAM tori for $1 D$ nonlinear wave equations with periodic boundary conditions, Comm. Math. Phys. 211 (2000), no. 2, 497-525.

[5] L. Corsi and R. Montalto, Quasi-periodic solutions for the forced Kirchhoff equation on $\mathbb{T}^{d}$, Nonlinearity 31 (2018), no. 11, 5075-5109.

[6] Y. Gao, W. Zhang and S. Ji, Quasi-periodic solutions of nonlinear wave equation with x-dependent coefficients, Internat. J. Bifur. Chaos Appl. Sci. Engrg. 25 (2015), no. 3, 1550043, $24 \mathrm{pp}$.

[7] J. Geng and X. Ren, Lower dimensional invariant tori with prescribed frequency for nonlinear wave equation, J. Differential Equations 249 (2010), no. 11, 2796-2821.

[8] S. B. Kuksin, Nearly integrable infinite-dimensional Hamiltonian systems, Lecture Notes in Mathematics 1556, Springer-Verlag, New York, 1993.

[9] R. Montalto, Quasi-periodic solutions of forced Kirchhoff equation, NoDEA Nonlinear Differential Equations Appl. 24 (2017), no. 1, Art. 9, 71 pp.

[10] J. Pöschel, A KAM-theorem for some nonlinear partial differential equations, Ann. Scuola Norm. Sup. Pisa Cl. Sci. (4) 23 (1996), no. 1, 119-148.

[11]_, Quasi-periodic solutions for a nonlinear wave equation, Comment. Math. Helv. 71 (1996), no. 2, 269-296.

[12] _ A lecture on the classical KAM theorem, in: Smooth Ergodic Theory and its Applications (Seattle, WA, 1999), 707-732, Proc. Sympos. Pure Math. 69, Amer. Math. Soc., Providence, RI, 2001.

[13] J. Si, Quasi-periodic solutions of a non-autonomous wave equations with quasiperiodic forcing, J. Differential Equations 252 (2012), no. 10, 5274-5360.

[14] Y. Wang, Quasi-periodic solutions of a quasi-periodically forced nonlinear beam equation, Commun. Nonlinear Sci. Numer. Simul. 17 (2012), no. 6, 2682-2700.

[15] C. E. Wayne, Periodic and quasi-periodic solutions of nonlinear wave equations via KAM theory, Comm. Math. Phys. 127 (1990), no. 3, 479-528.

[16] X. Yuan, Quasi-periodic solutions of completely resonant nonlinear wave equations, J. Differential Equations 230 (2006), no. 1, 213-274.

[17] _ Quasi-periodic solutions of nonlinear wave equations with a prescribed potential, Discrete Contin. Dyn. Syst. 16 (2006), no. 3, 615-634. 
[18] M. Zhang and J. Si, Quasi-periodic solutions of nonlinear wave equations with quasiperiodic forcing, Phys. D 238 (2009), no. 22, 2185-2215.

Yi Wang

School of Mathematics and Quantitative Economics, Shandong University of Finance and Economics, Jinan, Shandong 250014, P. R. China

E-mail address: yiwang8080@126.com

Jie Rui

College of Science, China University of Petroleum (East China), Qingdao, Shandong 266580, P. R. China

E-mail address: rjhygl@163.com 